九州大学学術情報リポジトリ

Kyushu University Institutional Repository

\title{
On hydrodynamic characteristics of gap resonance between two fixed bodies in close proximity
}

Gao, Junliang

School of Naval Architecture and Ocean Engineering, Jiangsu University of Science and Technology

Zang, Jun

Research Unit for Water, Environment and Infrastructure Resilience (WEIR), Department of Architecture and Civil Engineering, University of Bath

Chen, Lifen

Faculty of Engineering and Mathematical Sciences, University of Western Australia

Chen, Qiang

Research Unit for Water, Environment and Infrastructure Resilience (WEIR), Department of Architecture and Civil Engineering, University of Bath

他

http://hdl. handle. net/2324/4055218

出版情報：0cean Engineering. 173，pp. 28-44，2019-02-01. Elsevier バージョン：

権利関係: Creative Commons Attribution NonCommercial NoDerivatives 4.0 International 
On hydrodynamic characteristics of gap resonance between two fixed bodies in close proximity Junliang Gao ${ }^{1,2}$, Jun Zang ${ }^{2}$, Lifen Chen ${ }^{3}$, Qiang Chen ${ }^{2}$, Haoyu Ding ${ }^{2}$, Yingyi Liu ${ }^{4}$ 1 School of Naval Architecture and Ocean Engineering, Jiangsu University of Science and Technology, Zhenjiang 212003, China

2. Research Unit for Water, Environment and Infrastructure Resilience (WEIR), Department of Architecture and Civil Engineering, University of Bath, BA2 7AY, U.K.

3. Faculty of Engineering and Mathematical Sciences, University of Western Australia, Crawley WA6009, Australia

4. Research Institute for Applied Mechanics, Kyushu University, Kasuga, Fukuoka 816-8580, Japan

\begin{abstract}
:
The resonant water motion inside a narrow gap between two identical fixed boxes that are in side-by-side configuration is investigated using a two-dimensional (2D) numerical wave tank based on OpenFOAM ${ }^{\circledR}$, an open source CFD package. Gap resonance is excited by regular waves with various wave heights, ranging from linear waves to strong nonlinear waves. This paper mainly focuses on the harmonic analyses of the free-surface elevation in the narrow gap and wave loads (including the horizontal wave forces, the vertical wave forces and the moments) on the bodies. It is found that the influences of the incident wave height on the higher-order harmonic components of different physical quantities are quite different. The effects of the incident wave height on the reflection, transmission and energy loss coefficients are also discussed. Finally, aiming at the quantitative estimation of the response time and the damping time of gap resonance, two different methods are proposed and verified for the first time on gap resonance.
\end{abstract}

Keywords: Gap resonance; Wave height amplification; Wave force; Harmonic analysis; Response time and damping time of gap resonance; OpenFOAM ${ }^{\circledR}$

\footnotetext{
*Corresponding author. E-mail: J.Zang@bath.ac.uk.
} 
1. Introduction

In the past few decades, as the oil and gas industry have moved towards deeper waters and harsher environments, Floating Production Storage and Offloading (FPSO) platforms have shown great potential as the most economic ways to process and distribute the hydrocarbon products. One of the key challenges for FPSO platforms lies in the safe offloading operations from them to a shuttle tanker when the tanker is positioned side-by-side with them. When multiple floating bodies are deployed side-by-side in close proximity and are subjected to incident water waves, drastic water surface oscillations may occur inside the narrow gaps between them at certain frequencies. This phenomenon is normally referred to as "gap resonance”. investigated extensively due to its relevance to offloading facilities for FPSO. The methods used in these studies include theoretical analyses, physical experiments and numerical simulations. The theoretical analyses were mainly used in the early studies of the gap resonance problem and were mainly based on the linear potential flow theory (Miao et al., 2000; Molin, 2001). Subsequently, to better understand gap resonance and to validate the theoretical analyses, a large number of physical model tests in 2D and 3D wave basins were also implemented by previous researchers (e.g., Iwata et al. (2007); Saitoh et al. (2006); Zhao et al. (2017)). The numerical investigations conducted so far are mainly based on the classical potential flow model employing the boundary element method and scaled boundary finite element method (e.g., Li et al. (2005); Li and Zhang (2016); Sun et al. (2010)).

Although both theoretical analyses and the numerical simulations based on the potential flow theory have been shown to predict the resonant frequency well, they were reported to significantly over-estimate the resonant wave height inside the gap and the wave forces on the floating bodies, because the physical energy dissipation due to the fluid viscosity, vortex shedding and even turbulences cannot be considered in the context of potential flow theory. To overcome this problem, several particular numerical techniques that artificially introduce wave energy dissipation term into the potential flow model were developed so far (Chen, 2004; Huijsmans et al., 2001; Lu et al., 2010b; Newman, 2004; Ning et al., 2015a, b). However, the introduction of artificial damping term seems somewhat arbitrary for the rigorous potential theory, and under some conditions it was found to be difficult to obtain a unique value of the damping parameter 
(Pauw et al., 2007; Tan et al., 2014). In recent years, with the fast developments of computing technology, the CFD simulation has gradually become an alternative method in investigating the gap resonance problem (Jiang et al. (2018); Lu et al. (2010a); Lu et al. (2011a); Lu et al. (2011b); Moradi et al. (2015, 2016)). All these studies found that the results obtained by the CFD simulations agreed well with those from existing experiments.

While many research efforts into the gap resonance have been undertaken, the majority have concentrated on the analyses of the overall resonant wave height in the narrow gap and the overall wave loads on the boxes under the condition of the linear or weakly nonlinear regular waves (e.g., Feng et al. (2017); Jiang et al. (2018); Lu et al. (2010a); Lu et al. (2010b); Lu et al. (2011a); Lu et al. (2011b); Moradi et al. (2015, 2016)). The investigations on the harmonic analyses of the wave height and wave loads are relatively rare. By using a semi-analytical formulation of the velocity potentials, Mavrakos and Chatjigeorgiou (2009) investigated the significance of the second-order effects to the total wave loading on a cylindrical moonpool, especially in the frequency regions in which the fluid resonance occurs. Sun et al. (2010) employed a 3D boundary element code DIFFRACT to investigate the first- and second-order loads and free-surface elevations for two side-by-side rectangular barges. However, both of their methods are based on the classical potential flow theory which does not consider the physical energy dissipation due to the viscous effect. Hence, some of their findings may not reflect real phenomena of the fluid resonance in the narrow gap or in the moonpool, where the physical energy dissipation plays an important role. Zhao et al. (2017) investigated experimentally the first and higher harmonic components of the resonant fluid response in the gap between two identical fixed rectangular boxes excited by the transient focused wave groups in a 3D wave basin. However, the gap resonance induced by the regular waves and the harmonic analyses on wave loads were not considered in that paper.

To further improve the understanding of related phenomena involved in gap resonance, this paper mainly focuses on the variations of the first and higher harmonic components of free-surface elevation inside the gap and wave loads on boxes with respect to the wave height of the incident regular waves when gap resonance occurs. In this paper, the system of two identical boxes is taken as the background of this study. For comparison, the same problem is also investigated when the free-surface elevation in the narrow gap is under non-resonant conditions. Compared to the previous investigations (i.e., Feng et al. (2017); Jiang et al. (2018); Lu et al. (2010a); Lu et al. 
incident waves are used in this paper, which is necessary due to the fact that FPSO platforms are often exposed to severe wave conditions. Subsequently, the variations of the reflection coefficient $C_{r}$, the transmission coefficient $C_{t}$ and the energy loss coefficient $L_{e}=1-C_{r}^{2}-C_{t}^{2}$ with respect to the frequency of the incident waves with various wave heights are also discussed, because an integral comprehension of these coefficients may promote the better understanding of the mechanism essence of the gap resonance (Jiang et al., 2018). Meanwhile, these previous studies were mainly concerned on the related hydrodynamic phenomena after the free-surface resonance in the narrow gap reached the steady state, and both the response and the damp phases were paid resonance are quantitatively evaluated by two different methods. In practical engineering applications, the fast and accurate estimation of the response time and the damping time is very important for the safe evacuation of staff and the reasonable arrangement of operation time during the offloading operations from a FPSO platform to a shuttle tanker under gap resonance conditions.

In Sections 2, 3 and 4, the numerical model employed in this work, numerical experimental setup and the validations of the numerical model against available experimental and numerical data are presented, respectively. The numerical results and discussions are presented in Section 5.

\section{Numerical model description}

To consider the physical energy dissipation near the gap due to the viscous effect, a viscous flow solver is necessary. In this paper, the numerical wave tank is based on the OpenFOAM ${ }^{\circledR}$ multiphase solver "interFoam”, and waves are generated and dissipated using the relaxation-based wave generation toolbox “waves2Foam” proposed by Jacobsen et al. (2012).

\subsection{Governing equations}

The continuity and Navier-Stokes equations are utilized as the governing equations to solve the two-phase flow of water and air:

$$
\frac{\partial \rho}{\partial t}+\nabla \cdot(\rho \mathbf{u})=0
$$


where $\rho$ is the fluid density, $\nabla=\left(\frac{\partial}{\partial x}, \frac{\partial}{\partial y}, \frac{\partial}{\partial z}\right)$ is the gradient operation, $\mathbf{u}=(u, v, w)$ is the velocity vector of the fluid, $\mathbf{x}=(x, y, z)$ is the Cartesian coordinate vector, $\mathbf{g}$ is the gravitational acceleration, $P$ is the pressure in excess of the hydrostatic part, $\mu$ is the dynamic viscosity of the fluid, $\sigma_{t}$ is the surface tension coefficient and $k_{\alpha}$ is the surface curvature. The above equations are solved for both water and air simultaneously. $\alpha$ denotes the volume fraction of water in the computational cell, which takes a value of 1 for water and 0 for air and intermediate values for a mixture of water and air. The distribution of $\alpha$ is modelled by the following advection transport equation:

$$
\frac{\partial \alpha}{\partial t}+\nabla \cdot(\alpha \mathbf{u})+\nabla \cdot\left[\alpha(1-\alpha) \mathbf{u}_{\mathrm{r}}\right]=0
$$

in which $\mathbf{u}_{\mathrm{r}}=\mathbf{u}_{\text {water }}-\mathbf{u}_{\text {air }}$ is a relative velocity between the water and the air. Using $\alpha$, the spatial variation of any fluid property $\varphi$ (e.g., the fluid density $\rho$ and the dynamic viscosity $\mu$ ) can be expressed through the weighting

$$
\varphi=\alpha \varphi_{\text {water }}+(1-\alpha) \varphi_{\text {air }},
$$

where the subscripts "water" and "air" denote the corresponding fluid property of water and air, respectively.

\subsection{Boundary conditions and numerical implementations}

The toolbox “waves2Foam” proposed by Jacobsen et al. (2012) is employed to generate and absorb waves at the boundaries (see Fig. 1). At the inlet and the outlet boundaries, the velocities are defined as that of a regular incoming wave and as zero, respectively, and the pressure gradients are set to zero. Two relaxation zones are deployed at the inlet and the outlet boundaries to absorb the reflected and the transmitted waves. At the upper part of the tank, the boundary condition is set as "atmosphere"; while at the bottom of the tank and the solid walls of the fixed boxes, "no-slip" boundary condition is applied. For a 2D problem, the boundary condition on the walls in the third dimension is set to "empty".

The governing equations (1)-(2) and the advection transport equation (3) are solved based on the finite volume method. The velocity-pressure coupling is calculated using the PISO (Pressure Implicit with Splitting of Operator) algorithm. Gradients are approximated by the Gaussian integration method based on a linear interpolation form cell centers to cell faces. The time 

used for the evaluation of the divergence terms. Identical to Feng et al. (2017), to produce accurate and stable results, the largest Courant number is set to 0.25 in all simulations.

Once Eqs. (1)-(3) are solved at each time step, the wave force and the moment on the structure can be calculated by the following formulations:

$$
\mathbf{F}=\int_{\Omega}\left[P \mathbf{n}+\mu\left(\partial \mathbf{u}_{\tau} / \partial \mathbf{n}\right)\right] d s,
$$

and

where $\mathbf{F}$ and $\mathbf{M}$ are the vectors of the wave force and the moment, respectively, $\mathbf{u}_{\tau}$ is the tangent velocity component, $\mathbf{n}$ is the unit normal vector, $d s$ is the surface area differential on the wet solid surface $\Omega$, and $\mathbf{r}$ is the position vector of $d s$ relative to a certain space point. For the gap resonance problem that will be described in detail in Section 3, the moments on the two fixed boxes correspond to their respective centroids. As for the harmonic analysis for various variables (i.e., the free-surface elevation in the gap, the horizontal and vertical wave forces and the moments on their respective signals.
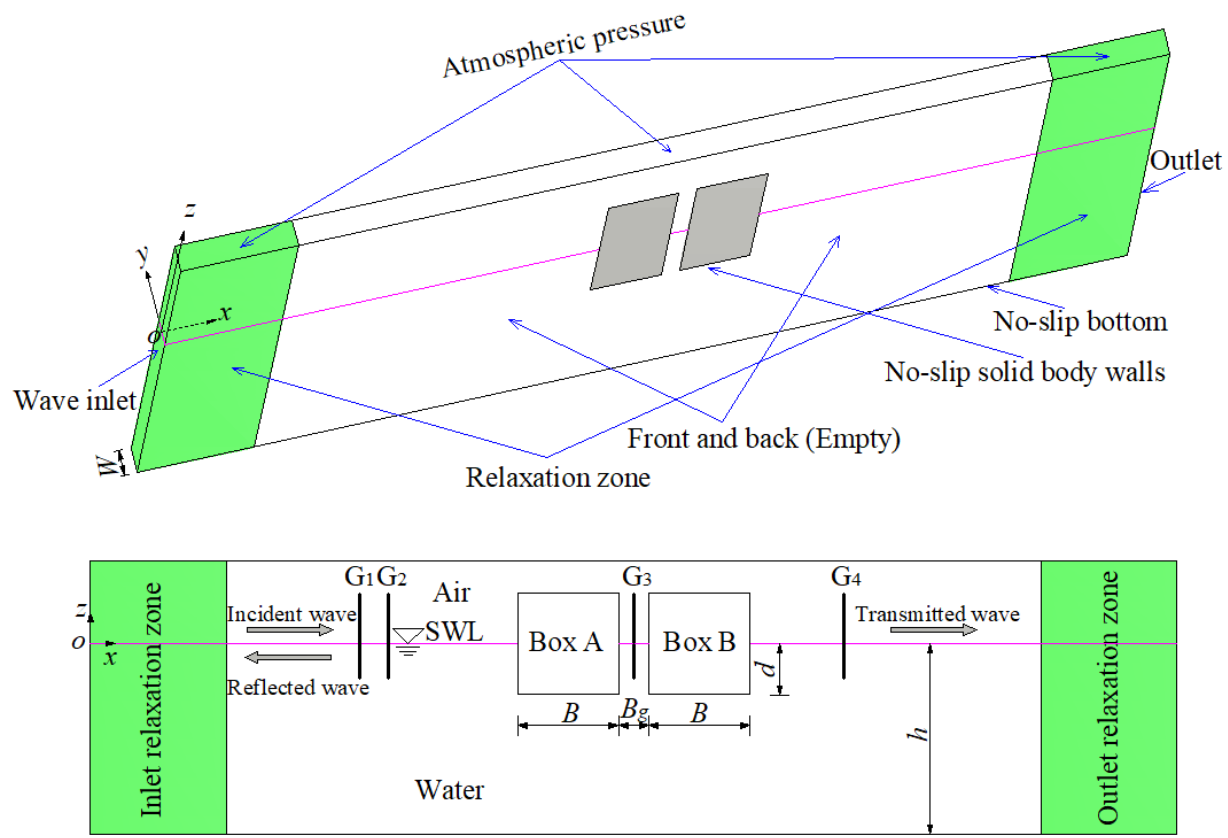

Fig. 1. Sketch of the numerical wave tank: (a) boundary conditions and the definition of the coordinate system; (b) positions of wave gauges and the definition of the geometric parameters. 


\section{Numerical wave tank}

Fig. 1 illustrates the sketch of the 2D numerical wave tank used in the present study. The wave tank has a length of $18.5 \mathrm{~m}$, a height of $0.8 \mathrm{~m}$ and a width of $W=0.1 \mathrm{~m}$. The origin of the coordinate system is located at the still water level (SWL) of the left inlet boundary. The $x$-axis is in the wave propagation direction, and the $z$-axis is in the upward direction. The thickness of the wave tank in $y$-direction corresponds to a cell. Two identical fixed boxes are placed at the middle of the wave tank. The box height is $H=0.5 \mathrm{~m}$, the breadth is $B=0.5 \mathrm{~m}$, the draft $d=0.25 \mathrm{~m}$, the gap width $B_{g}=0.05 \mathrm{~m}$, the water depth is $h=0.5 \mathrm{~m}$, and the air depth is $h_{a}=0.3 \mathrm{~m}$. This configuration is in accordance with the physical experiments in Saitoh et al. (2006) as well as the numerical investigations in Lu et al. (2008; 2011a; 2011b).

Five sets of simulations are implemented, in which the wave heights of the incident regular waves are set to $H_{0}=0.010 \mathrm{~m}, 0.024 \mathrm{~m}, 0.050 \mathrm{~m}, 0.075 \mathrm{~m}$ and $0.100 \mathrm{~m}$, respectively. The wave frequency, $\omega$, considered in all the five sets of simulations ranges from $4.456 \mathrm{rad} / \mathrm{s}$ to $7.534 \mathrm{rad} / \mathrm{s}$. Correspondingly, the dimensionless wavenumber, $k h$, ranges from 1.210 to 2.910 , where $k=2 \pi / L$ denotes the wavenumber and $L$ denotes the wavelength. Four wave gauges, $\mathrm{G}_{1}-\mathrm{G}_{4}$, are arranged to record the free-surface elevations. $G_{1}$ and $G_{2}$ are utilized to decompose the incident and reflected waves, and their distance is set to $0.25 \mathrm{~m} . \mathrm{G}_{3}$ and $\mathrm{G}_{4}$ are used to obtain the free surfaces inside the gap and the transmitted waves. $G_{3}$ is placed in the middle of the gap; while $G_{2}$ and $G_{4}$ are positioned at $1.50 \mathrm{~m}$ from the left side of Box A and the right side of Box B, respectively. Two relaxation zones of $5.50 \mathrm{~m}$ long each are placed at the inlet and outlet boundaries of the wave tank to absorb the reflected and transmitted waves. The length of $5.50 \mathrm{~m}$ is approximately 2.11 times of the maximum wavelength that corresponds to the incident waves with $\omega=4.456 \mathrm{rad} / \mathrm{s}$.

A built-in mesh generation utility supplied with OpenFOAM ${ }^{\circledR}$, “blockMesh”, is employed to generate meshes. A typical computational mesh is shown in Fig. 2. Non-uniform meshes are adopted for saving the computational time. The fine meshes with higher resolution are used around the boxes, especially in the vicinity of the narrow gap. To capture the interface between water and air, the meshes gradually become denser from the bottom and the atmosphere boundaries to the still water level. 


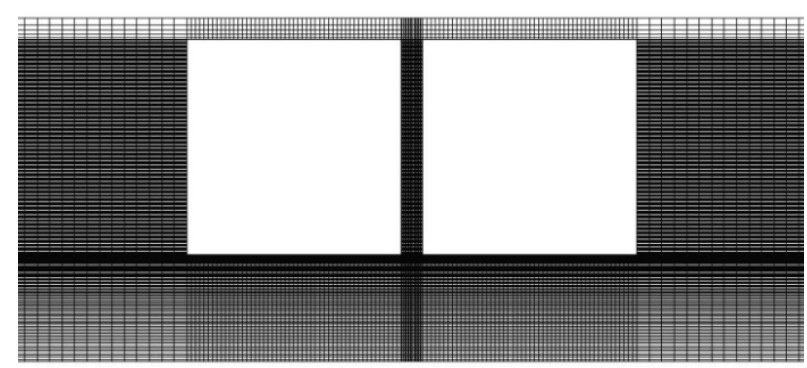

201

Fig. 2. Side view of typical meshes in the computational domain: (a) the meshes around the boxes;

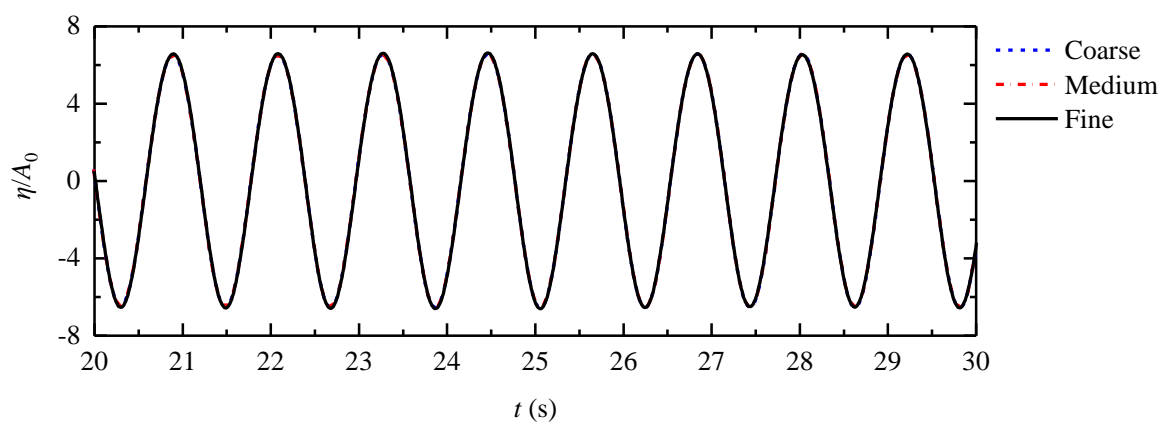

Fig. 3. Dependence of the free-surface elevation in the gap on the mesh resolution for the incident waves with $k h=1.556$ and $H_{0}=0.010 \mathrm{~m}$, in which $A_{0}=H_{0} / 2$ denotes the amplitude of the incident waves.

To examine the dependence of the numerical results on the mesh resolution, the free-surface response in the narrow gap is simulated using three different meshes, namely the coarse, medium and fine meshes. The numbers of the cells for these three meshes are 143600, 224060 and 340880, respectively. Based on the numerical results that will be shown in Section 4.1, the free-surface resonance in the gap occurs at $k h=1.556$. Fig. 3 presents the resonant free surfaces inside the gap induced by the incident waves with $k h=1.556$ and $H_{0}=0.010 \mathrm{~m} . A_{0}=H_{0} / 2$ in this figure denotes the incident wave amplitude. It is seen that the time histories of the free-surface elevations for the 
three mesh configurations are almost identical to each other. Meanwhile, considering that the medium mesh can provide more accurate simulations of the wave fields excited by the incident waves with higher frequencies as compared to the coarse mesh, in all our numerical simulations, the medium mesh configuration is employed.

\section{Numerical model validations}

To guarantee the reliability of the model and the accuracy of the numerical results, the numerical model and the numerical wave tank illustrated in Sections 2 and 3 are first validated by comparing the present results obtained by OpenFOAM ${ }^{\circledR}$ with available experimental data and numerical results in previous literatures. For the simulations with $H_{0}=0.024 \mathrm{~m}$ described in Section 3, Saitoh et al. (2006) and Lu et al. (2011b) have measured the amplification of the free-surface elevation inside the gap and the wave forces on boxes by using physical experiments and a viscous flow model, respectively. Comparisons of the present results with those in the two papers will be presented in Section 4.1. Because the current research mainly focuses on the harmonic analysis of the free-surface elevation in the gap and the wave loads on the boxes, it is essential to further examine the capability of the present model to predict the higher-order harmonic components of the free-surface elevation or the wave loads. To the best of our knowledge, for the gap resonance problem, the experimental data on the higher-order harmonic components of the free-surface elevation or the wave loads are rare. However, Rodríguez et al. (2016) implemented physical experiments on the interactions between regular waves and one fixed box, and the experimental data of the vertical wave force on the box (including the first- and 


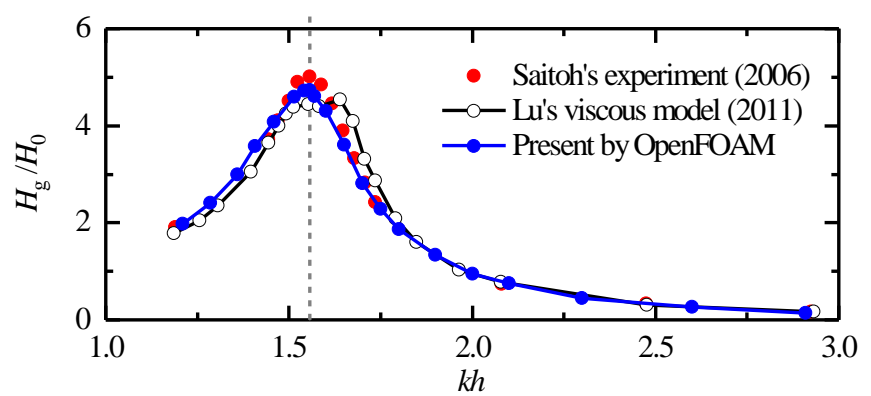

Fig. 4 illustrates the amplification of the free-surface elevation inside the narrow gap excited by the incident waves with $H_{0}=0.024 \mathrm{~m}$. It can be seen that the predicted resonant frequency, $k h=1.556$, by the present numerical model is almost identical to those obtained by both the laboratory tests of Saitoh et al. (2006) and the CFD results of Lu et al. (2011b). Besides, in general, the variation of $H_{\mathrm{g}} / H_{0}$ with respect to $k h$ also agrees well with their results. Fig. 5 further presents the comparisons of the horizontal and vertical wave forces on Boxes A and B predicted by OpenFOAM $^{\circledR}$ and those by the CFD results in Lu et al. (2011b). Similar to Fig. 4, the overall agreement between the present results and those in Lu et al. (2011b) is also observed.
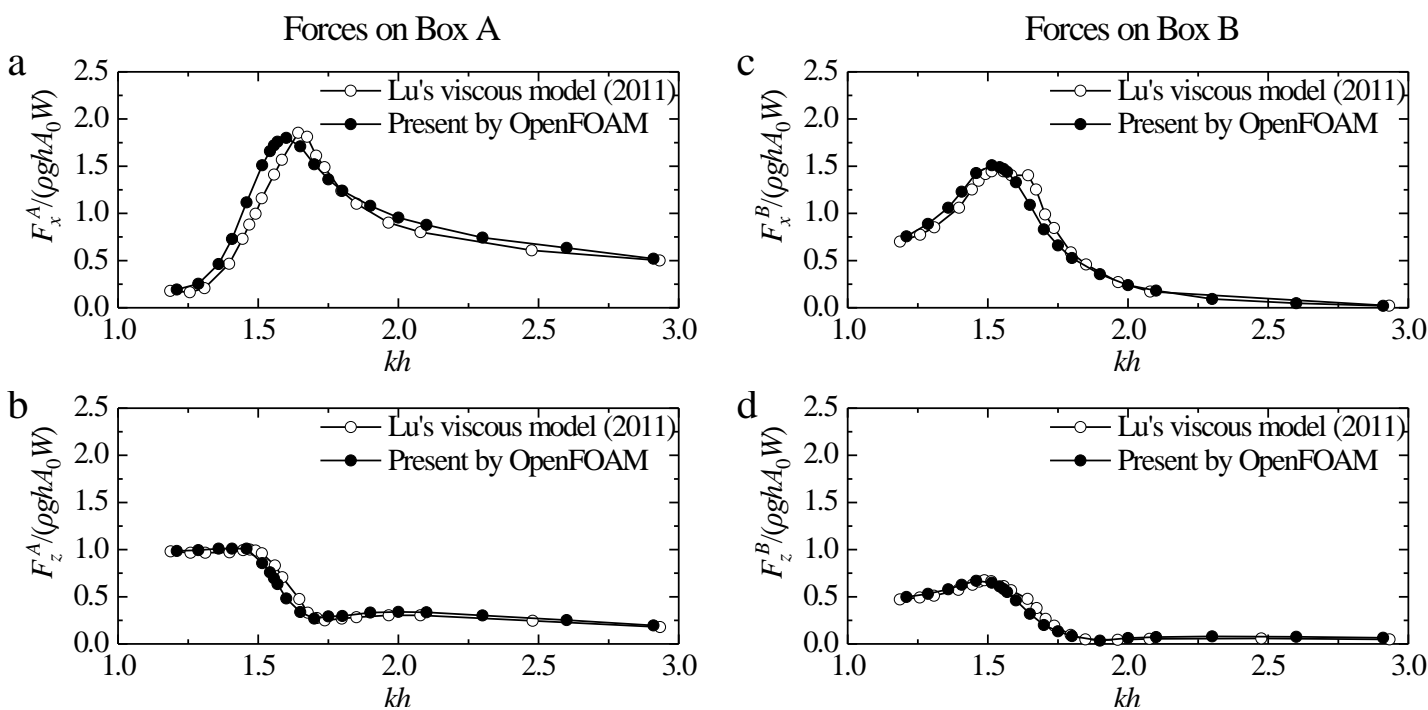

Fig. 5. Variations of the wave forces on the two boxes with respect to the incident wave frequency. 

correspond to the horizontal and vertical forces on Box B, respectively.

\subsection{One-box condition}

Rodríguez et al. (2016) performed laboratory experiments in a 2.79 m wide and $63.00 \mathrm{~m}$ long wave tank, and the water depth is $h=1.25 \mathrm{~m}$. A rectangular box was placed approximately in the center of the wave tank, at $x=29 \mathrm{~m}$, where $x=0$ defines the location of the wave-maker. Because the experimental study sought to achieve 2D flow conditions, the width of the rectangular box was chosen as $2.76 \mathrm{~m}$, leaving only a very small gap of $0.015 \mathrm{~m}$ to either of the tank's sidewalls. Single box geometry with the breadth $B=0.50 \mathrm{~m}$ and the draught $d=0.25 \mathrm{~m}$. The regular incident waves with $0.4 \leq k B \leq 2.4$ were considered. Two series of physical experiments were carried out with two steepnesses of the incident waves $k A_{0}=0.05$ and 0.10 . To examine the performance of the numerical model for the strongly nonlinear wave conditions, the series of experiments with $k A_{0}=$ 0.10 are reproduced by OpenFOAM ${ }^{\circledR}$ here. Considering that the box used in Rodríguez et al. (2016) has the same breadth and draft with the two boxes shown in Fig. 1, a very similar numerical wave tank (not shown in this paper for brevity) with that in Fig. 1 is employed to implement the present simulations. Compared to the wave tank shown in Fig. 1, there only exist two main differences in the present wave tank. First, there is only a single box located in the middle of the present wave tank. Second, the water depth is deepened from $0.50 \mathrm{~m}$ to $1.25 \mathrm{~m}$. A mesh configuration that has a similar mesh density with the medium mesh described in Section 3 is utilized. It should be noted that due to the relaxation zone deployed around the inlet and outlet boundaries, it is not necessary for the numerical wave tank to set the same length, $63.00 \mathrm{~m}$, as the physical wave tank, and the numerical tank with a length of $18.5 \mathrm{~m}$ is already long enough.

Fig. 6 presents the simulated and experimental time-histories of the non-dimensional vertical wave force, $F_{z}(t) /\left(0.5 \rho g A_{0} B W\right)$, for three cases with $k B=0.8,1.4$, and 2.0 . It can be obviously seen that significant nonlinearities are present, particularly for $k B=1.4$ and 2.0 , due to the vertical asymmetry of the force traces. Overall, the agreement between the present numerical results and the experimental data is good. Fig. 7 further quantitatively compares the first- and second-order harmonic components of the experimental and numerical vertical forces for all cases with $k A_{0}=0.10$. Good agreement between the experimental and numerical results is also observed. 
$\mathrm{a}$

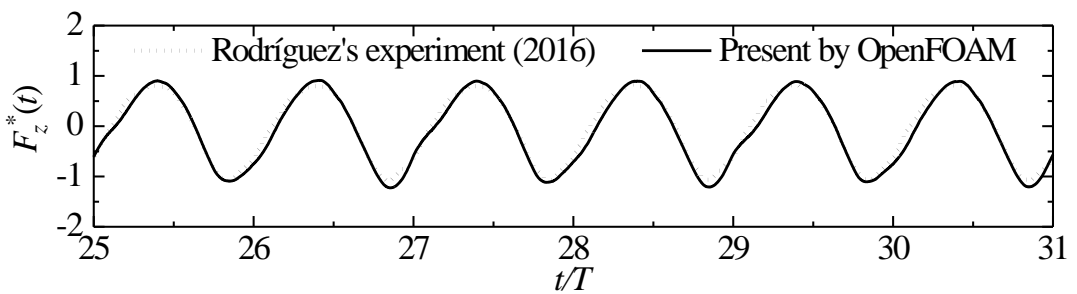

b

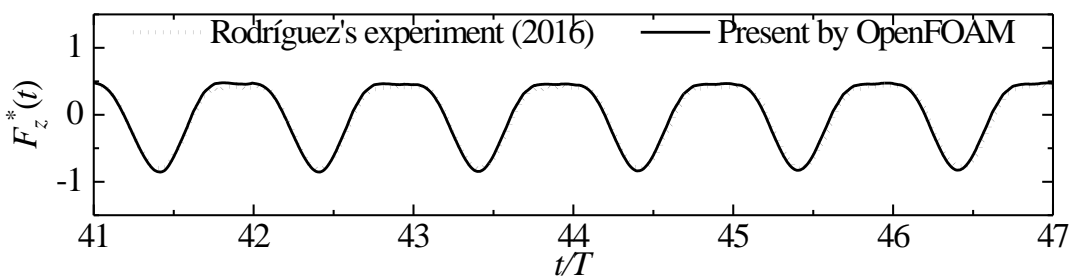

$\mathrm{C}$

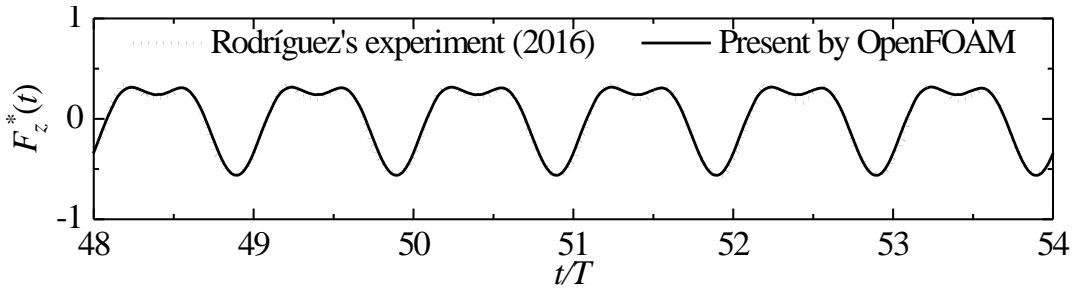

Fig. 6. Time histories of the vertical wave forces excited by the regular waves with $k A_{0}=0.10$ and (a) $k B=0.8$, (b) $k B=1.4$ and (c) $k B=2.0$, in which $F_{z}{ }^{*}(t)=F_{z}(t) /\left(0.5 \rho g A_{0} B W\right)$ denotes the time history of the non-dimensional vertical wave forces.

302
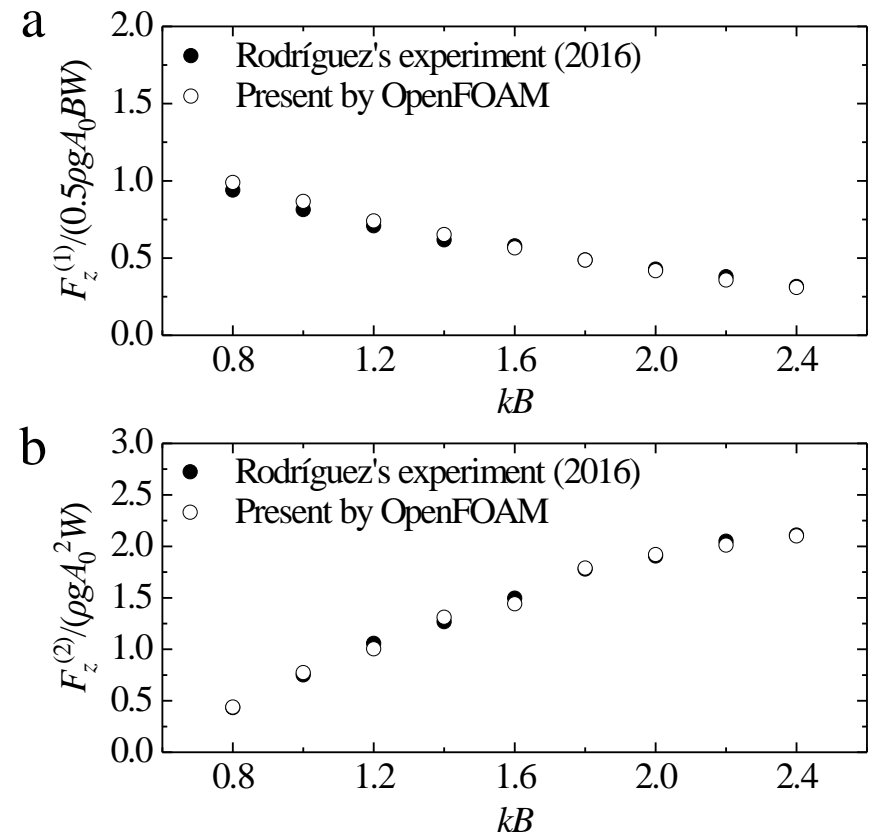

Fig. 7. Non-dimensional (a) first-order and (b) second-order vertical wave forces excited by the incident regular waves with $k A_{0}=0.10$ 
5. Numerical results and discussion

In order to present an overall impression of the hydrodynamic characteristics of gap resonance under the conditions of various incident wave heights on the reader, the variations of the overall wave height amplification in the narrow gap and the overall wave loads on boxes with respect to the incident wave frequency are first illustrated and discussed in Section 5.1. Subsequently, to find out the relative importance of different harmonic components, the first three order harmonic components of the free-surface elevation in the narrow gap and the wave loads on boxes are analyzed in Section 5.2. Then, to better explain some phenomena presented in Sections 5.1 and 5.2 and better understand the mechanism essence of gap resonance, the variations of the reflection, transmission and energy loss coefficients with respect to the frequency of the incident waves with various wave heights are discussed in Section 5.3. Finally, considering the importance of the fast and accurate estimation of the response time and the damping time of gap resonance, two different estimation methods are proposed and verified in Section 5.4.

\subsection{Overall wave height amplifications and overall wave loads}

Fig. 8 shows the overall free-surface amplification in the narrow gap and the overall wave forces and moments impacting on Boxes A and B excited by the incident regular waves with various wave heights. Four obvious phenomena can be easily seen. First, it is seen from Fig. 8a that the resonant frequency seems not sensitive to the incident wave height. For the cases with $H_{0}=0.010 \mathrm{~m}, 0.024 \mathrm{~m}$ and $0.100 \mathrm{~m}$, all the three variation curves of the free-surface amplification with the frequency present perfect single-peak shapes, and the maximum free-surface amplification in the narrow gap always occurs at the resonant frequency, i.e., $k h=1.556$. However, for the cases with $H_{0}=0.050 \mathrm{~m}$ and $0.075 \mathrm{~m}$, the two variation curves of the free-surface amplification do not show the perfect single-peak shape. The two curves around the resonant frequency become flat, and the values of free-surface amplification at $k h=1.556$ are even slightly less than the ones at its both adjacent sides. The reason for this phenomenon can be attributed to the almost invariable reflection coefficients around the resonant frequency under the conditions of $H_{0}=0.050 \mathrm{~m}$ and $0.075 \mathrm{~m}$ (it will be shown in Section 5.3). 

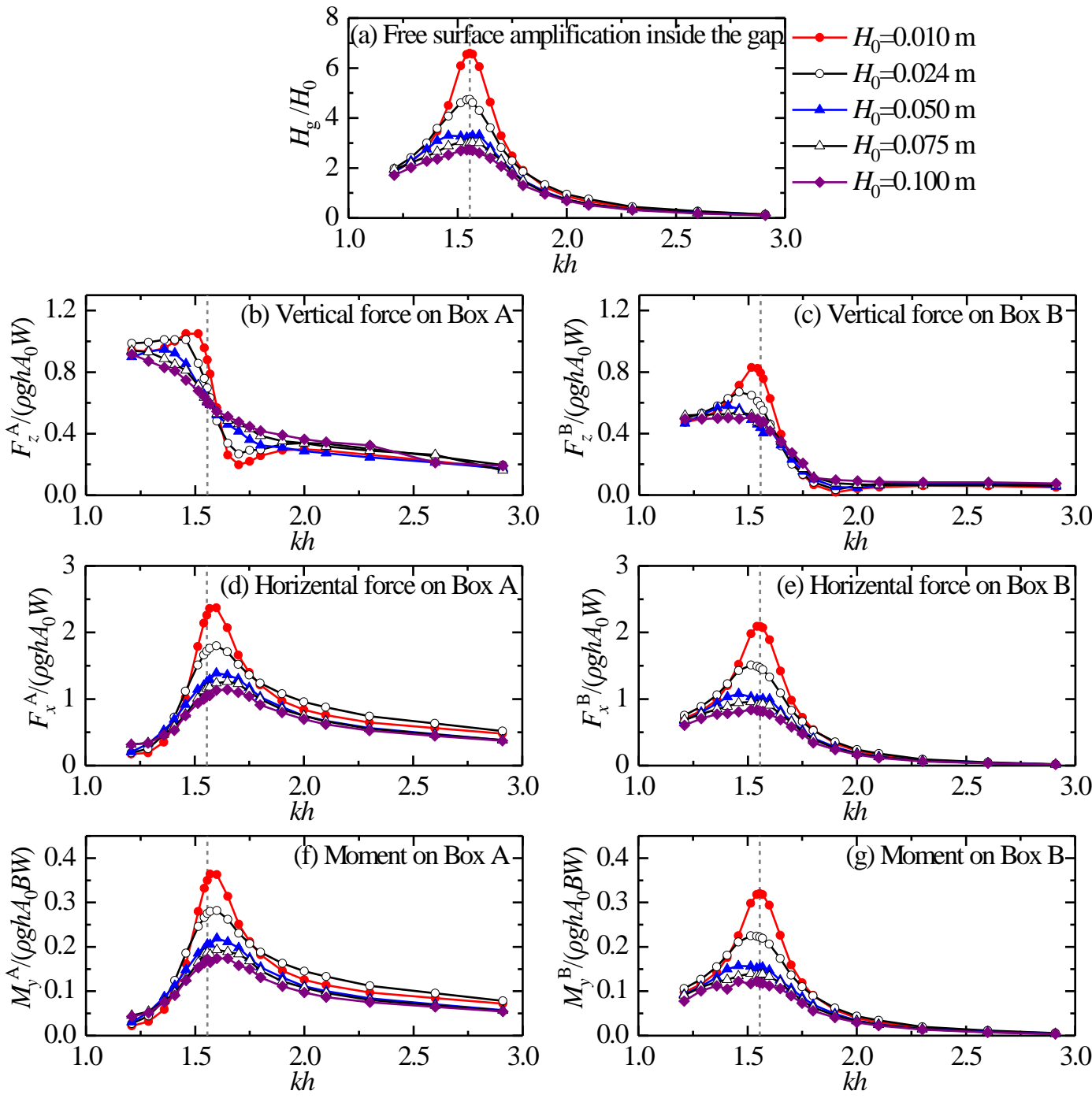

Fig. 8. The overall free surface amplification in the gap and the overall wave forces and moments on Boxes A and B induced by the incident regular waves with various wave heights. The vertical dash line refers to the position of the resonant frequency.

Second, for the vertical wave forces on both the two boxes (Fig. 8b and c), there exist obvious deviations between the frequency at which the maximum vertical wave force appears and the resonant frequency. However, there are some different features for the changing trends of the vertical wave forces on the two boxes. For Box A, the difference between the frequency at which the maximum vertical wave force appears and the resonant frequency monotonously increases with the incident wave height. Besides, the vertical wave forces excited by the incident wave with small height tends to increase first, then sharply decrease, then slowly increase, and then slowly decrease with the non-dimensional wavenumber, $k h$. However, with the increase of the incident 
wave height, the vertical wave forces gradually become monotonic decrease with $k h$. For Box B, when the incident wave height is small, the changing trend of the vertical wave forces with $k h$ is similar to that for Box A. When the incident wave height becomes large, the value of the vertical wave force seems insensible to the incident wave frequency at the ranges of $k h<1.5$ and $k h>1.9$, and its value only decreases sharply with the incident wave frequency at the range of $1.5<k h<$ 1.9.

Third, for the horizontal wave forces on Box A (Fig. 8d), the frequency at which the maximum horizontal force occurs is obviously larger than the resonant frequency; the larger the incident wave height is, the more obvious the deviation becomes. While for the horizontal wave forces on Box B (Fig. 8e), the frequency at which the maximum horizontal force occurs is equal to or just slightly less than the resonant frequency. It is due to the fact that the magnitude of the horizontal force is determined by the free-surface elevation difference between the opposite sides of the each box (Lu et al., 2011b). The free-surface elevation at the left side of Box A is much larger than that at the right side of Box B. It leads to that the free-surface elevation difference between the opposite sides of Box A is more different from the free-surface elevation in the gap, while the free-surface elevation difference between the opposite sides of Box B is more close to the free-surface elevation in the gap. As for the reason why the free-surface elevation at the left side of Box A is much larger than that at the right side of Box B, there are two main reasons: (1) the reflected wave height is always larger than the transmitted wave height (i.e., $C_{r}>C_{t}$, which will be shown in Section 5.3), and (2) the left side of Box A locates at a antinode of the partially standing waves composed of the incident and the reflected waves, which causes the wave height at the left side of Box A is approximately equal to the summation of the incident and the reflected wave heights.

Fourth, for the moments on Boxes A and B (Fig. 8f and g), for all the incident wave heights considered in this paper, the variation curves of the moment on each box with the frequency is very similar to those of the horizontal force on the corresponding box. Hence, the phenomena described above for the horizontal forces are also applicable for the moments. To further examine the phenomenon that the variation curves of the moment on each box with the frequency are very similar to those of the horizontal force on the corresponding box for all the incident wave heights studied in this paper, Fig. 9 presents the comparisons of the normalized curves of the horizontal 

refers to the original variation curve divided by the corresponding peak value of the original variation curve. Hence, the normalized curve always has a maximum value, 1.0. It can be seen that for both the two boxes and for both the two incident wave heights, the normalized curves of the horizontal wave forces are almost identical to those of the moments. For the other three incident wave heights (i.e., $H_{0}=0.024 \mathrm{~m}, 0.050 \mathrm{~m}$ and $0.075 \mathrm{~m}$ ), the similar phenomenon can also be clearly observed (their comparisons are not shown in the paper for brevity).
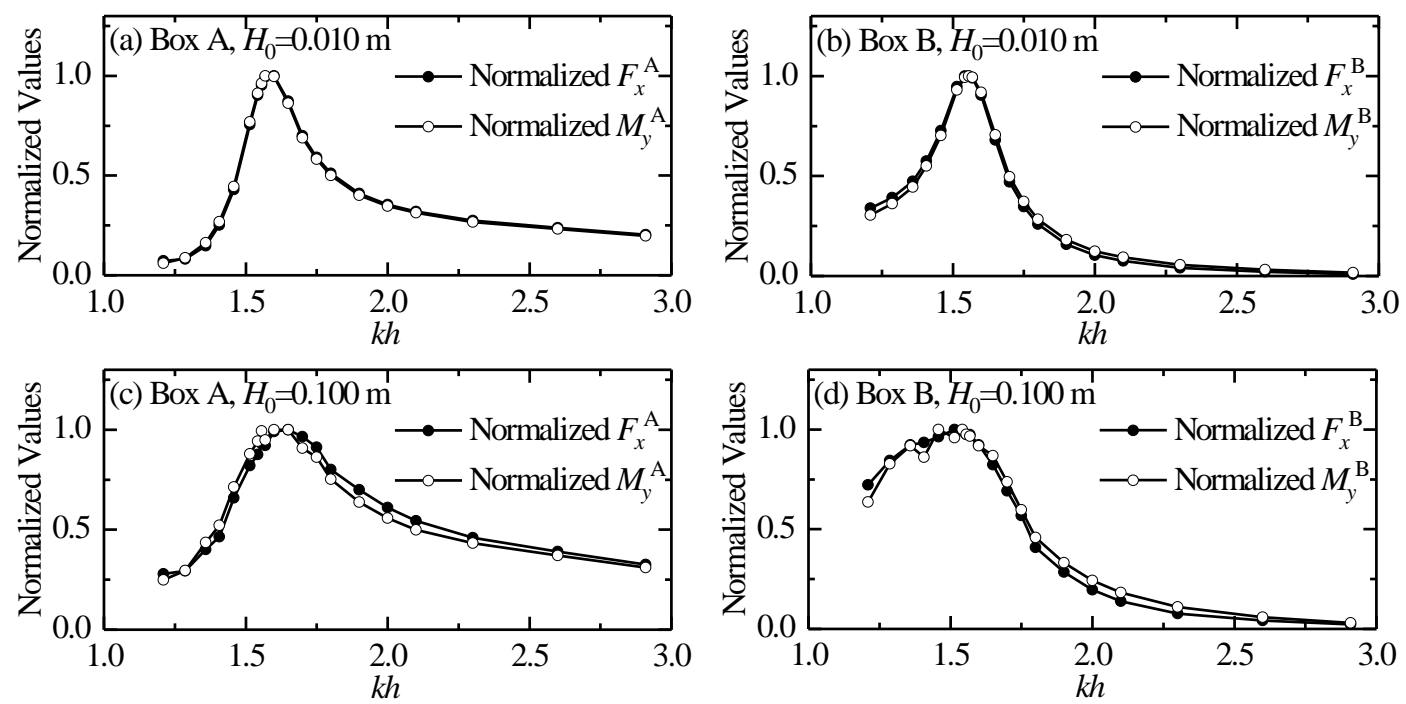

Fig. 9. Comparisons of the normalized curves of the horizontal wave forces and the moments on Boxes $\mathrm{A}$ and $\mathrm{B}$

5.2 Harmonic analyses of wave height amplifications and wave loads

Fig. 10 illustrates the variation of the first three order harmonic components of the free-surface elevation in the gap with respect to the frequency under the conditions of various incident wave heights. $H_{\mathrm{g}}^{(i)}(i=1,2$ and 3$)$ in the figure denotes the $i^{\text {th }}$-order harmonic component of the free-surface elevation in the gap. The following three phenomena can be easily observed. First, the first-order component of the free-surface elevation is significantly larger than the secondand third-order components. Second, around the resonant frequency, the second-order component is larger than the corresponding third-order one; the larger the incident wave height is, the more obvious the phenomenon becomes. Third, all the first three order harmonic components around the resonant frequency are remarkably larger than the corresponding ones for the non-resonant conditions. 
To quantify the relative importance of higher-order components to the first-order component, Fig. 11 further shows the ratios of the second- and third-order harmonic components to the first-order harmonic components for the free-surface elevation in the gap under the conditions of various incident wave heights. It is seen that at the range of $1.3<k h<1.9$, there existing obvious peak points around the resonant frequency for both the second- and third-order harmonic components. For the second-order harmonic components, the maximum of their ratios to the first-order harmonic components reaches up to about 13\%. It can be attributed to the fact that the free-surface elevation around the resonant frequency is remarkably amplified, and naturally the higher-order harmonic components of the free-surface elevation are enhanced due to the wave nonlinearity. While at the ranges of $k h<1.3$ and $k h>1.9$, as the wave frequency becomes far from the resonant frequency, the ratios of the second- and third-order components to the first-order components tend to gradually increase. It is mainly due to that the value of the first-order component significantly decreases as the wave frequency becomes far from the resonant frequency, especially for the high-frequency range (i.e., $k h>1.9$ ).
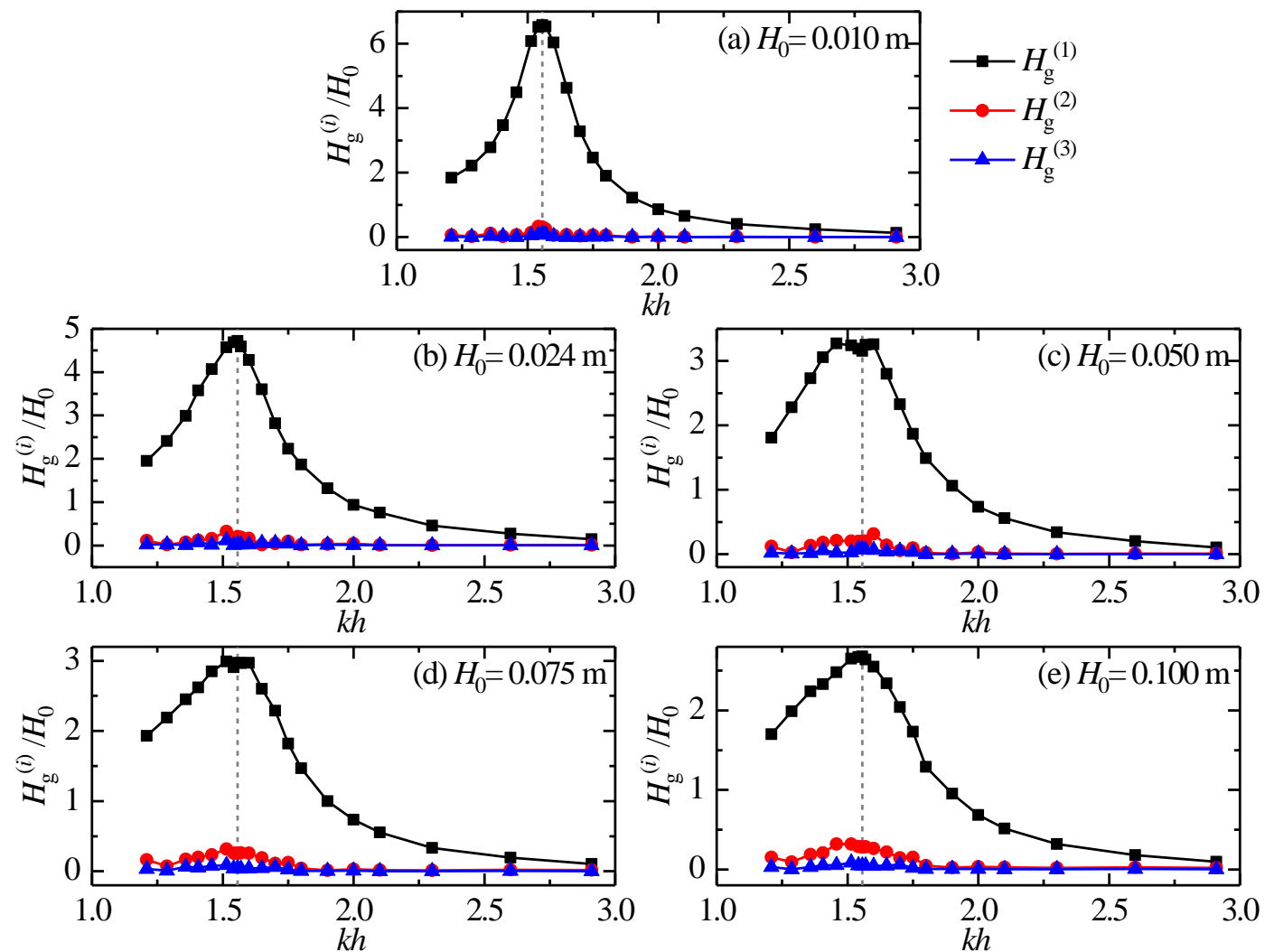

Fig. 10. The first three order harmonic components of the free-surface elevation in the gap under 


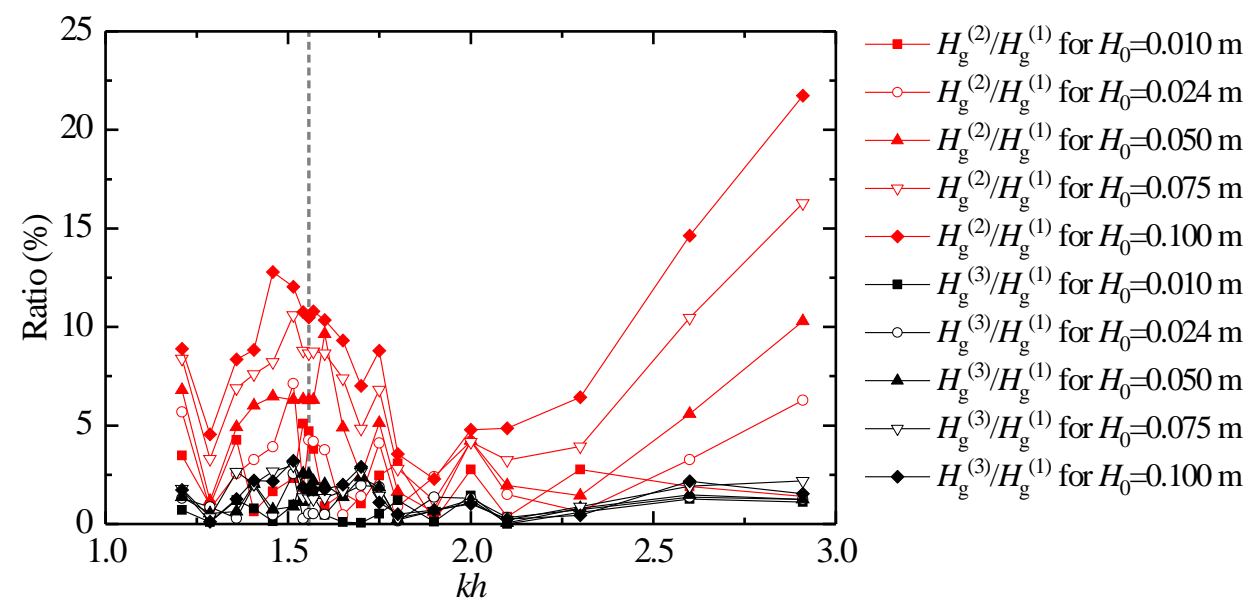

Fig. 12 illustrates the first three order harmonic components of the vertical wave forces on Boxes $\mathrm{A}$ and $\mathrm{B}$ for all the simulations. $F_{z}^{\mathrm{A}(i)}$ and $F_{z}^{\mathrm{B}(i)}(i=1,2$ and 3$)$ in this figure refer to the $i^{\text {th }}$-order harmonic components of the vertical wave forces on Boxes A and B, respectively. The following three phenomena can be easily seen. First, the first-order harmonic components of the vertical wave force are much larger than the higher-order components near the resonant frequency. Second, when the incident wave height is small (Fig. 12a and f), the second-order harmonic component is obviously larger than the third-order ones around the resonant frequency. As the incident wave height increases, the third-order harmonic components around the resonant frequency gradually become obviously larger than those far away from the resonant frequency; on the contrary, the second-order harmonic components around the resonant frequency become smaller and smaller compared with those far away from the resonant frequency. When the incident wave height increases up to $H_{0}=0.100 \mathrm{~m}$ (Fig. 12e and j), the third-order harmonic components have approached (for Box B) or even exceeded (for Box A) the second-order ones. Third, for the high-frequency range, because the first-order harmonic components decease sharply with the wave frequency, the second-order harmonic components approach and even exceed the corresponding first-order ones for both the two boxes.

Fig. 13 further shows the ratios of the second- and third-order harmonic components to the first-order harmonic components for the vertical wave forces on the two boxes for all the 
simulations. It can be easily observed that for both the two boxes and for the wave frequency far away from the resonant frequency, the ratio of the second- to the first-order components is always larger than the ratio of the third- to the first-order components, while around the resonant frequency, the latter approaches or even exceeds the former. Besides, for the second-order components, their ratios near the resonant frequency are less than those far from the resonant frequency. While for the third-order components, their ratios near the resonant frequency tend to be larger than those far from the resonant frequency (it is valid for the whole frequency range considered in this paper for Box A, and for $k h<1.800$ for Box B).

Vertical force on Box A
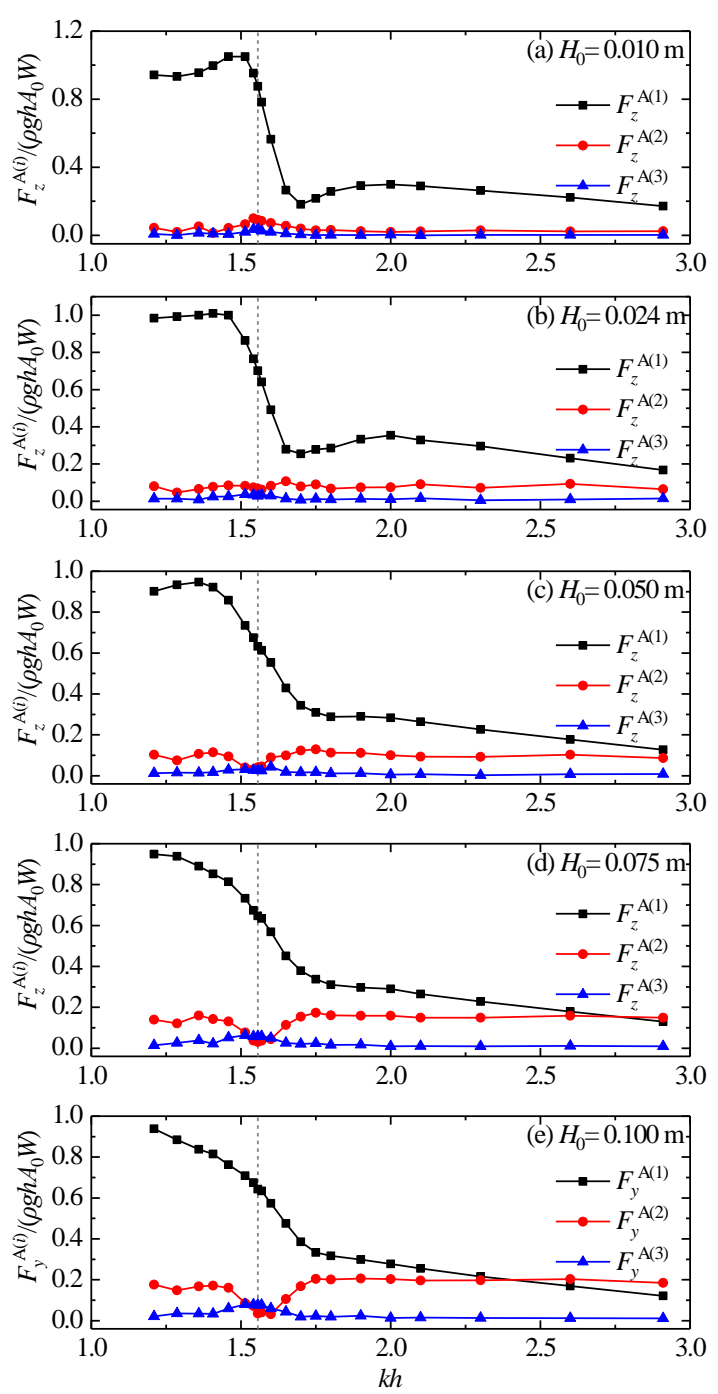

Vertical force on Box B
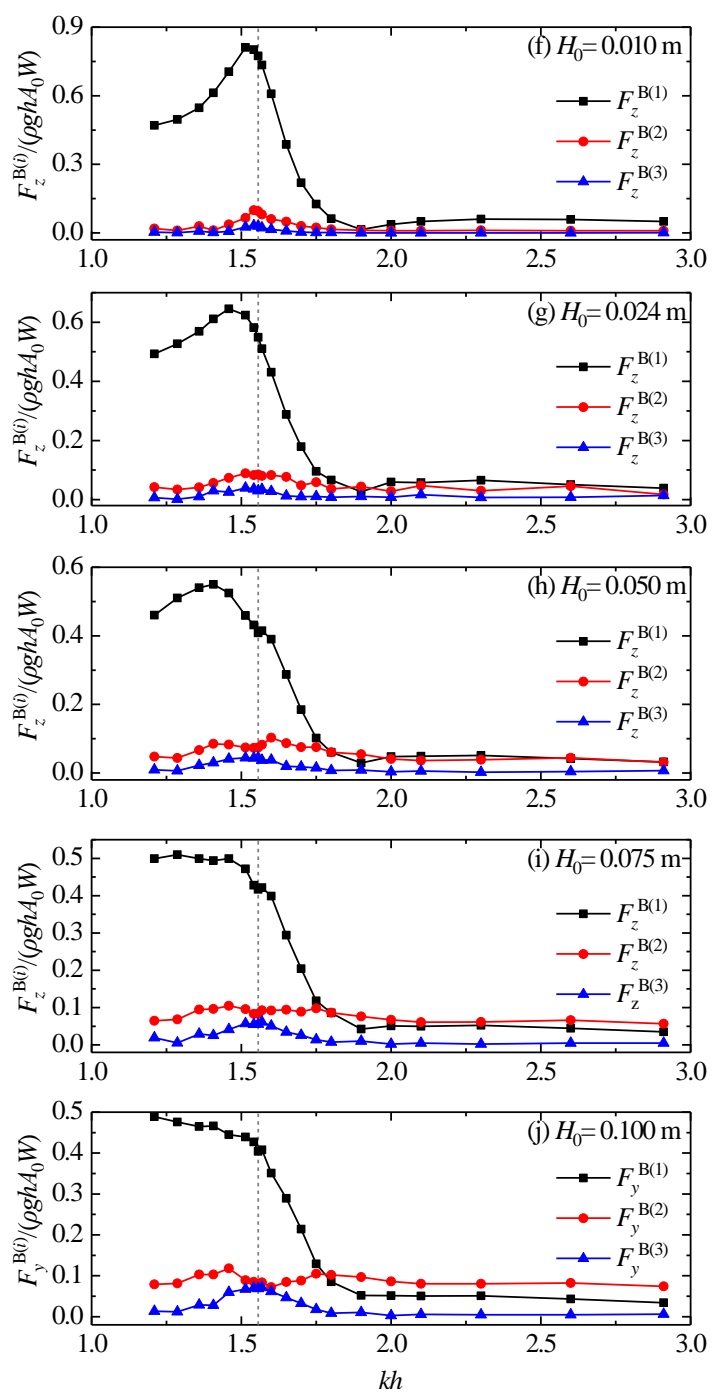

Fig. 12. The first three order harmonic components of the vertical wave forces on Boxes A and B under the conditions of various incident wave heights. 
(a)

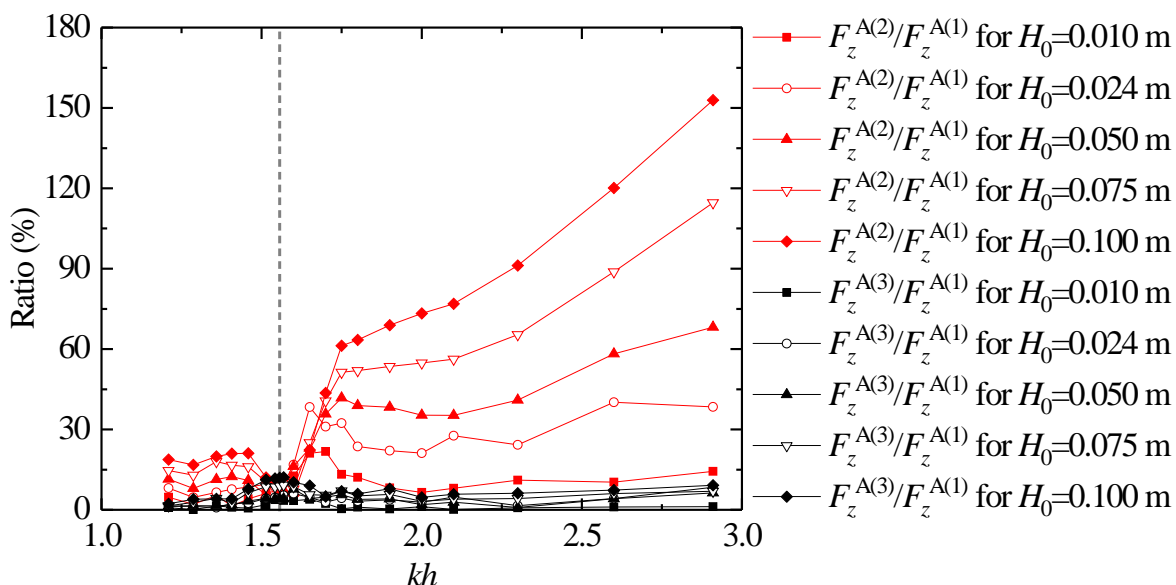

(b)

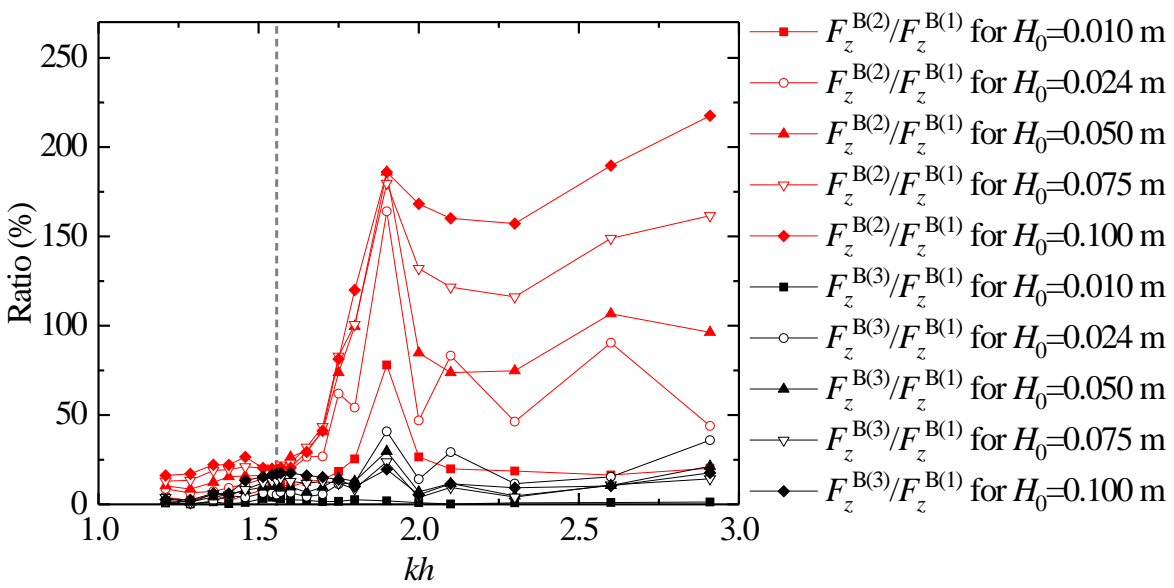

Fig. 13. Ratios of the second- and third-order harmonic components to the first-order harmonic components for the vertical wave forces on (a) Box A and (b) Box B under the conditions of various incident wave heights

Fig. 14 presents the first three order harmonic components of the horizontal wave forces on Boxes $\mathrm{A}$ and $\mathrm{B}$ for all the simulations. $F_{x}^{\mathrm{A}(i)}$ and $F_{x}^{\mathrm{B}(i)}(i=1,2$ and 3$)$ in this figure refer to the $i^{\text {th }}$-order harmonic components of the horizontal wave forces on Boxes A and B, respectively. Because both the second- and third-order harmonic components of the horizontal wave forces around the resonant frequency are extremely small compared to the corresponding first-order components, in order to better show the variations of all these three harmonic components with the incident wave frequency, the values of both the second- and third-order harmonic components shown in this figure are enlarged five times. In general, the above three phenomena for the vertical wave forces shown in Fig. 12 can also be observed in this figure.

Fig. 15 further presents the ratios of the second- and third-order harmonic components to the first-order harmonic components for the horizontal wave forces on the two boxes for all the 
simulations. It should be noted that, for Box B (Fig. 15b), when $k h=2.910$ and $H_{0}=0.100 \mathrm{~m}$, the value of $F_{x}^{\mathrm{B}(2)} / F_{x}^{\mathrm{B}(1)}$ has already exceeded $140 \%$. However, to better show the variation characteristics of the ratios around the resonant frequency, the maximum changing range of the $y$-axis is only set to $20 \%$. Again, in general, all the phenomena for the vertical wave forces presented in Fig. 13 can also be observed in this figure, except that the ratios of the third- to the those far from the resonant frequency.

Horizontal force on Box A
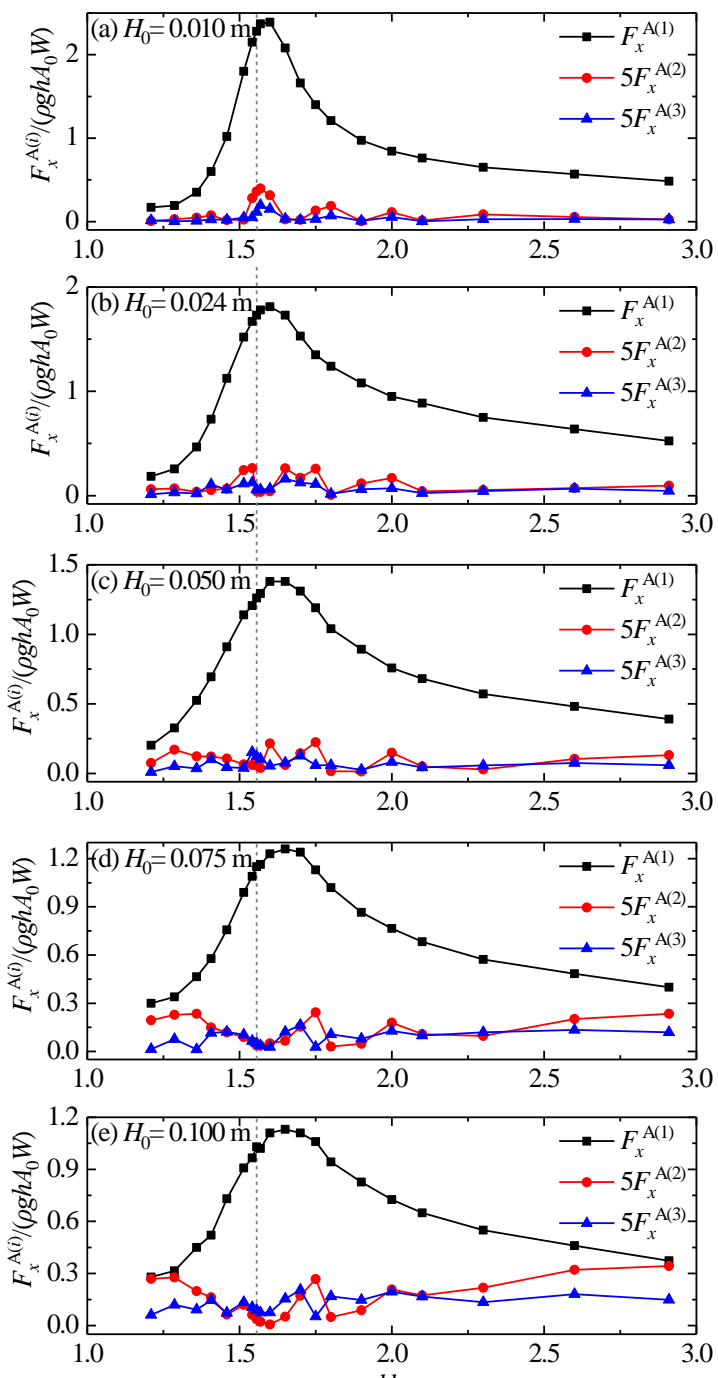

Horizontal force on Box B
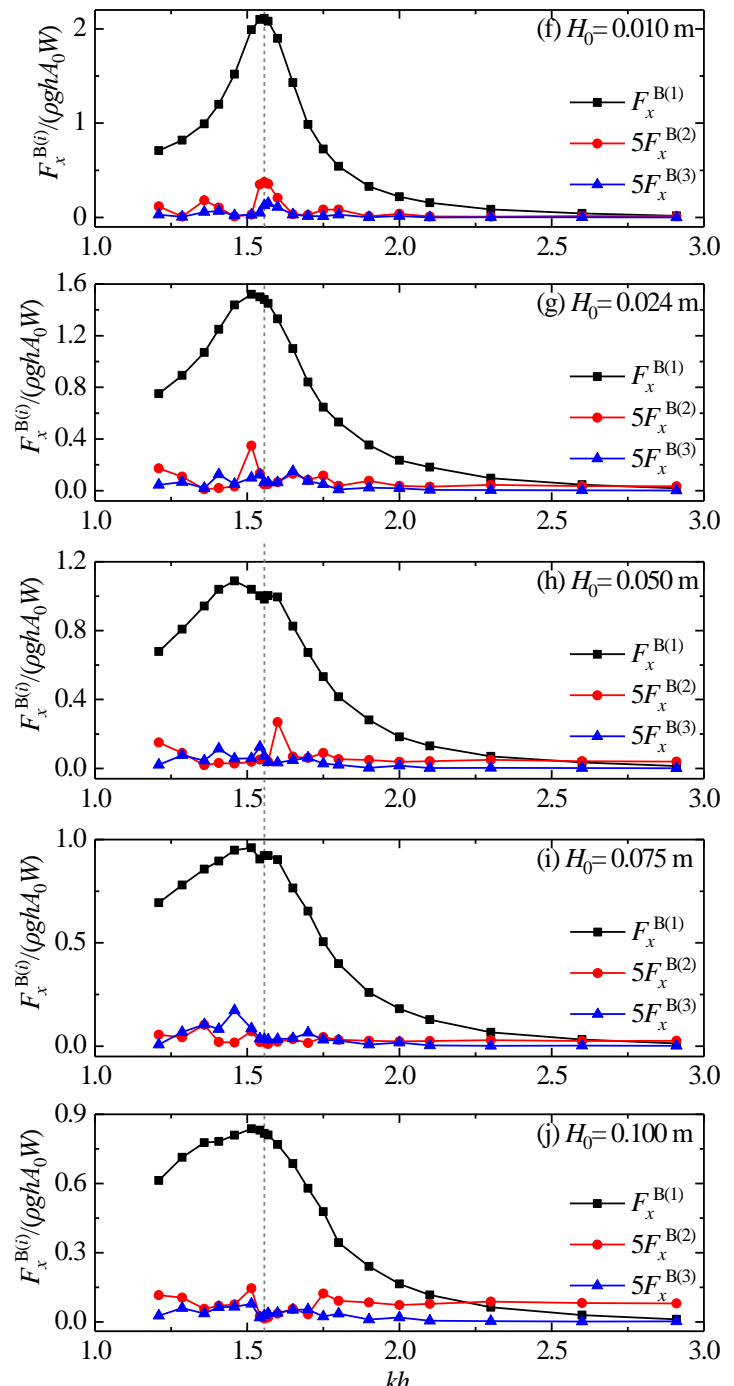

Fig. 14. The first three order harmonic components of the horizontal wave forces on Boxes A and $\mathrm{B}$ under the conditions of various incident wave heights. 
(a)

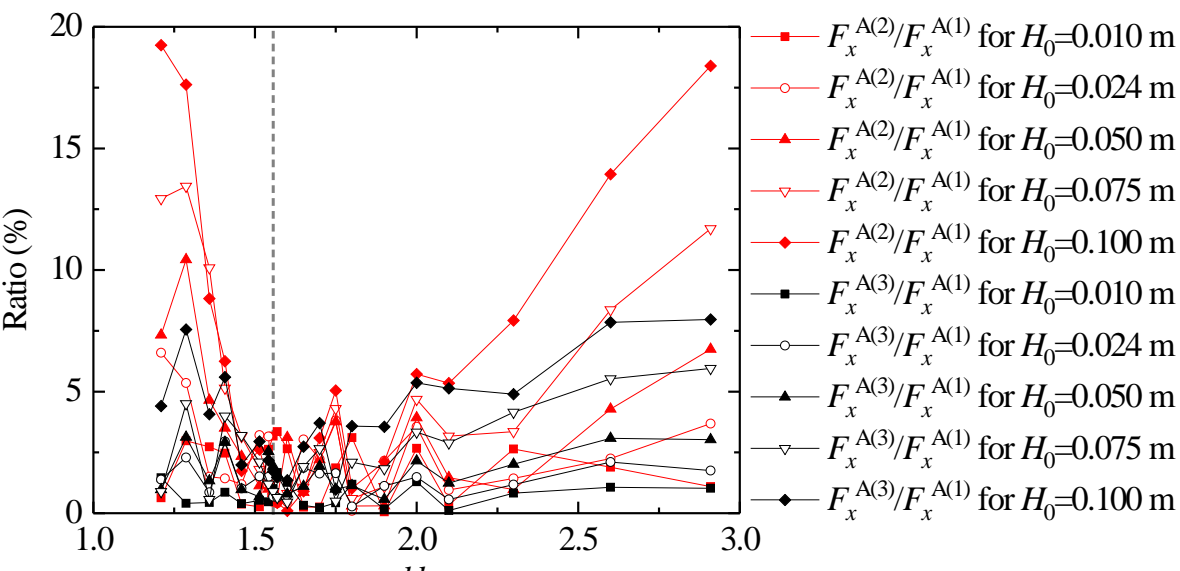

(b)

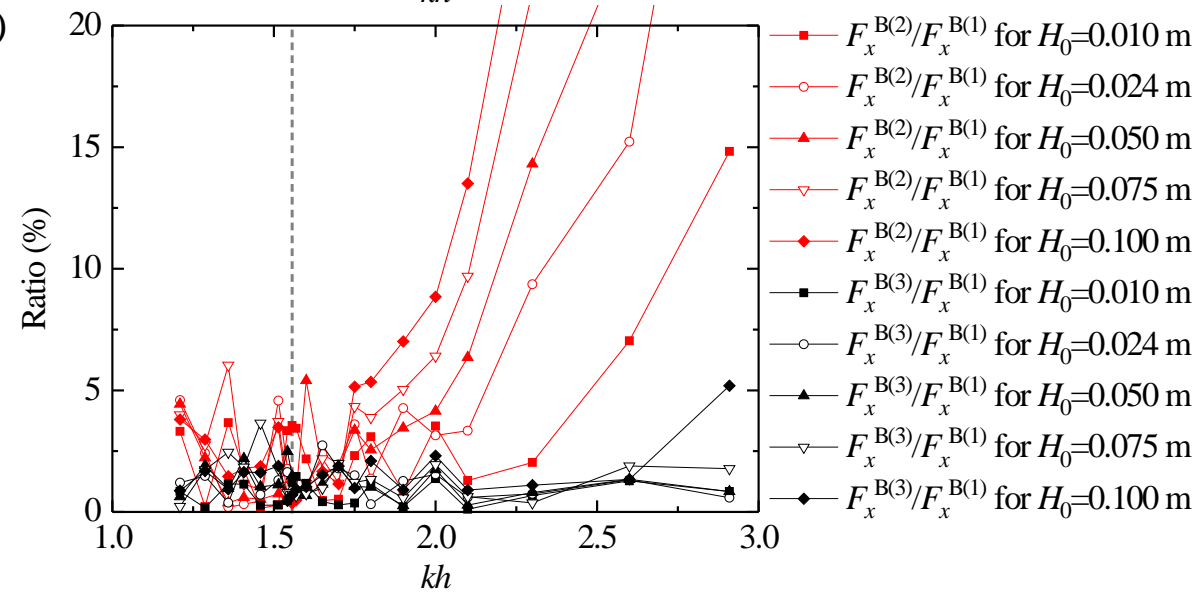

Fig. 15. Ratios of the second- and third-order harmonic components to the first-order harmonic components for the horizontal wave forces on (a) Box A and (b) Box B under the conditions of various incident wave heights 
Fig. 16 presents the first three order harmonic components of the moments on Boxes A and B

498 for all the simulations, in which $M_{y}^{\mathrm{A}(i)}$ and $M_{y}^{\mathrm{B}(i)}\left(i=1,2\right.$ and 3) refer to the $i^{\text {th }}$-order harmonic 499 components of the moments on Boxes A and B, respectively. It is obviously seen that when the 500 incident wave height is small (refer to Fig. 16a and f), both the second- and third-order harmonic 501 components are much smaller than the corresponding first-order ones around the resonant 502 frequency for both the two boxes. However, as the incident wave height increases, the values of 503 the second-order harmonic components around the resonant frequency gradually increase. 504 Compared to the first-order harmonic components, the second-order harmonic components have 505 reached a considerable values when $H_{0}=0.100 \mathrm{~m}$ (refer to Fig. 16e and j). To better illustrate this 506 point, the ratios of the second- and third-order harmonic components to the first-order harmonic 507 components for the moments on both the two boxes under the conditions of various incident wave 508 heights are presented in Fig. 17. It can be seen that when the incident wave height is small (i.e., 509 $\left.H_{0}=0.010 \mathrm{~m}\right)$, both the values of $M_{y}^{\mathrm{A}(2)} / M_{y}^{\mathrm{A}(1)}$ and $M_{y}^{\mathrm{B}(2)} / M_{y}^{\mathrm{B}(1)}$ are approximately $5 \%$. 510 However, when the incident wave height increases to $H_{0}=0.100 \mathrm{~m}$, both their values reach up to near $20 \%$. 
Moment on Box A
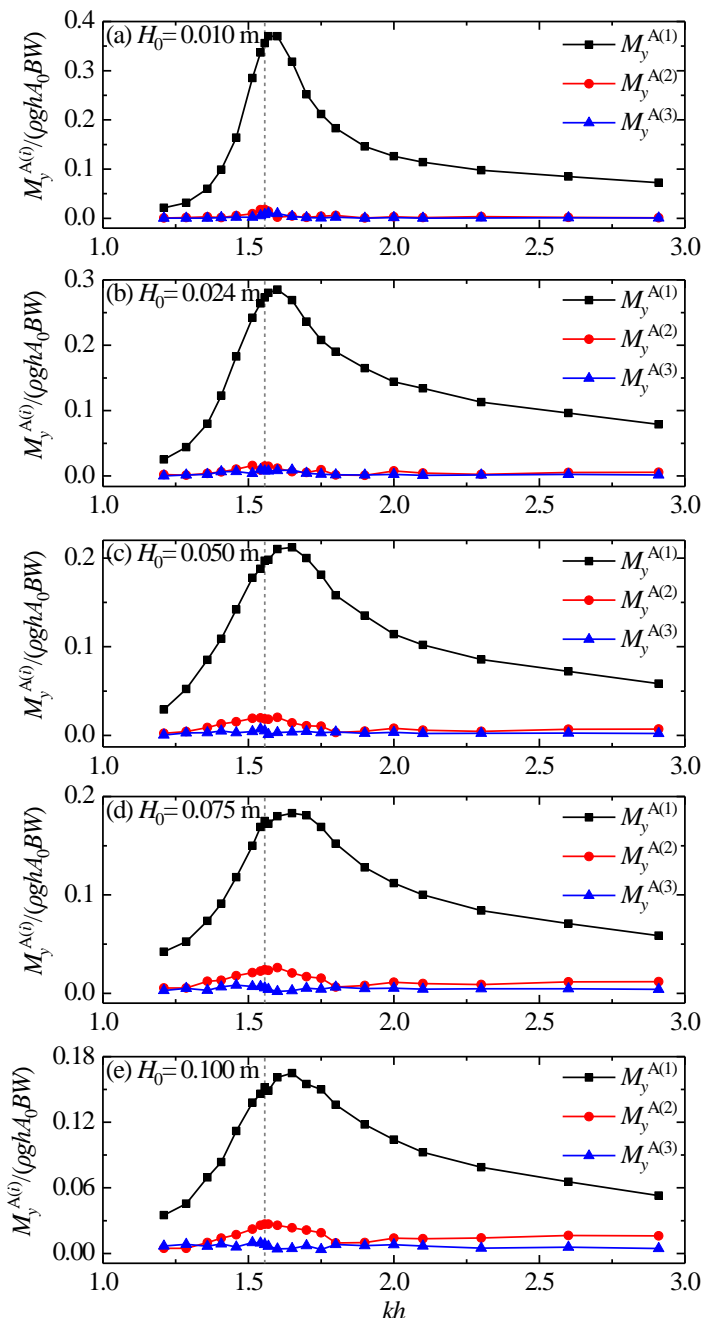

Moment on Box B
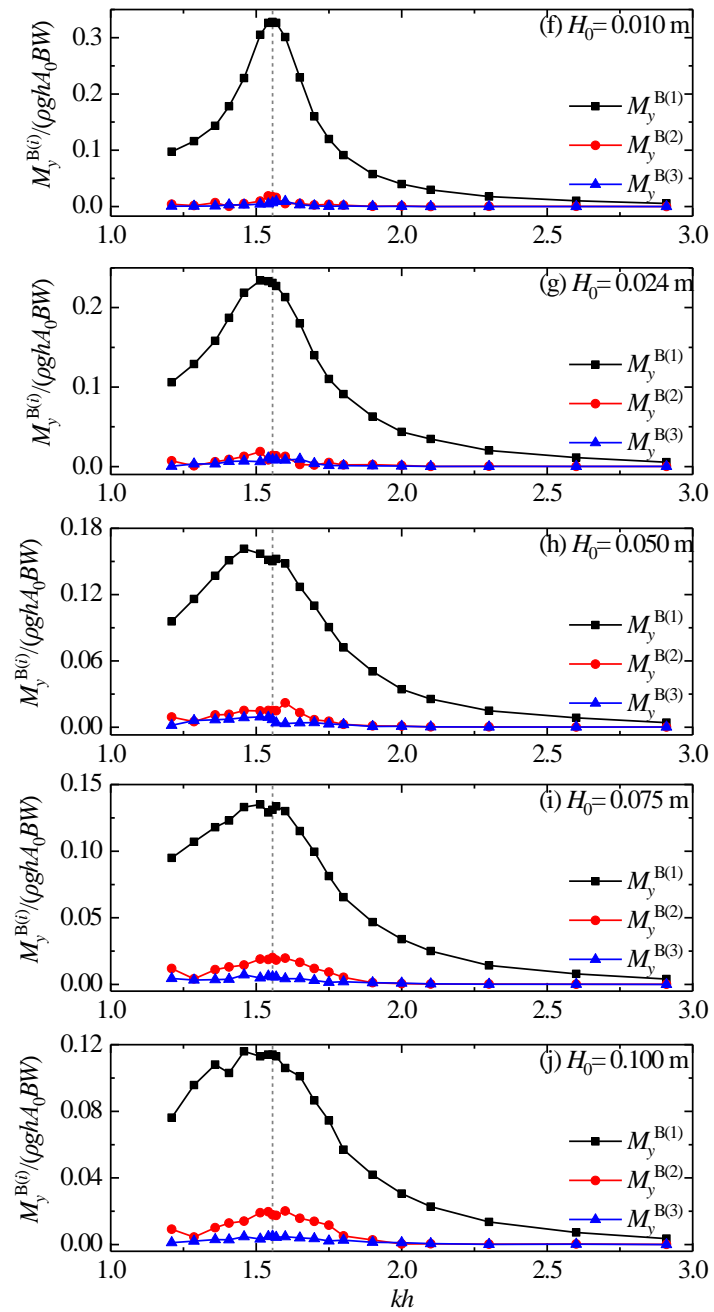

Fig. 16. The first three order harmonic components of the moments on Boxes A and B under the conditions of various incident wave heights. 
(a)

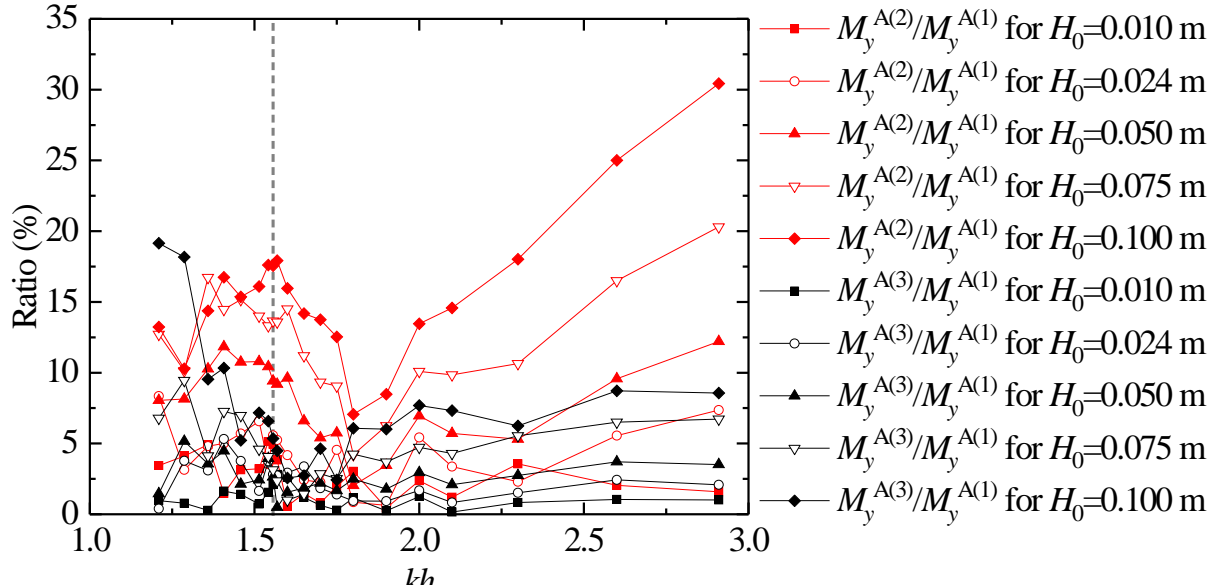

(b)

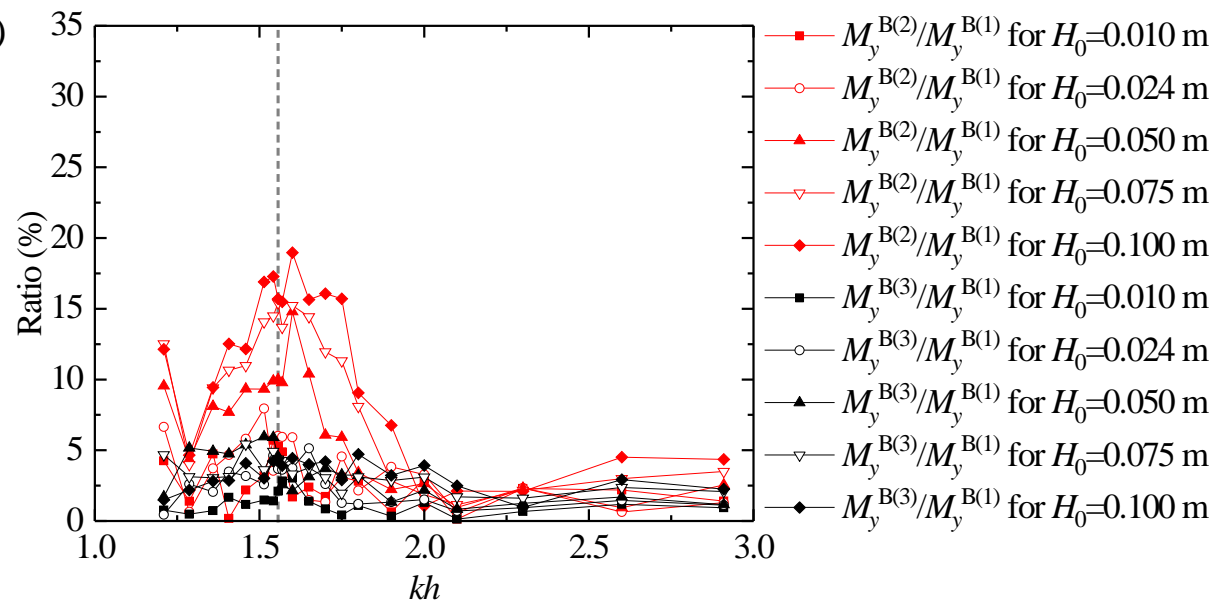

Fig. 17. Ratios of the second- and third-order harmonic components to the first-order harmonic components for the moments on (a) Box A and (b) Box B under the conditions of various incident wave heights

5.3 Reflection, transmission and energy loss coefficients

Based on the wave analysis technique in Goda and Suzuki (1976), the wave height of the reflected waves from the two-box system can be obtained by using the free-surface elevations at $\mathrm{G}_{1}$ and $\mathrm{G}_{2}$ (refer to Fig. 1). The reflection coefficient $C_{r}$ is further calculated as the ratio of the reflected wave height to the incident wave height $H_{0}$. The transmission coefficient $C_{t}$ is defined as the ratio of the transmitted wave height to $H_{0}$, and the transmitted wave height can be obtained by the free-surface elevation at $\mathrm{G}_{4}$. Then, the energy loss coefficient $L_{e}=1-C_{r}^{2}-C_{t}^{2}$ is calculated. The effects of the incident wave height on the reflection, transmission and energy loss coefficients, $C_{r}, C_{t}$ and $L_{e}$, are illustrated in Fig. 18. For the reflection coefficient (Fig. 18a), the following three phenomena can be easily observed. First, the reflection coefficients $C_{r}$ near the resonant frequency 
are always less than those away from the resonant frequency. Second, the reflection coefficient at the resonant frequency increases with the increasing of the incident wave height. Third, under the conditions of $H_{0}=0.050 \mathrm{~m}$ and $0.075 \mathrm{~m}$, both the two variation curves of the reflection coefficient around the resonant frequency almost become flat, which indicates that the similar wave energy

can propagate into the gap. Hence, this leads to the relatively flat variation curves of $H_{\mathrm{g}} / H_{0}$ around the resonant frequency for $H_{0}=0.050 \mathrm{~m}$ and $0.075 \mathrm{~m}$ shown in Fig. 8a.

For the transmission coefficient (Fig. 18b), the frequency at which the maximum transmission coefficient $C_{t}$ occurs is always less than the resonant frequency. The larger the incident wave height is, the more obvious their difference becomes. When the incident wave height is small, the transmission coefficient first increases, then sharply decreases, then slowly increases, and then slowly decreases with the non-dimensional wavenumber, $k h$. However, with the increase of the incident wave height, the vertical wave forces gradually become monotonic decrease with $k h$. These effects of the incident wave height on the variation characteristics of the transmission coefficient are very similar to its effects on those of the vertical wave forces on Boxes A and B (see Fig. 8b and c).

By carefully comparing Fig. 18a and b, it can be found that for all the incident wave heights considered in this paper, the reflection coefficients are always larger than the transmission coefficient, no matter whether the gap resonance occurs or not. The larger the incident wave height is, the more obvious the difference between $C_{r}$ and $C_{t}$ becomes. This explains that phenomenon shown in Fig. 8d and e that for the horizontal wave forces on Box A, the frequency at which the maximum horizontal force occurs obviously deviates from the resonant frequency; while for the horizontal wave forces on Box $\mathrm{B}$, the frequency at which the maximum horizontal force occurs is approximately equal to the resonant frequency.

For the energy loss coefficient (Fig. 18c), it is seen that for all the incident wave heights considered in this paper, almost all the maximum energy loss coefficients appear at (or very close to) the resonant frequency. Besides, with the increase of the incident wave height, the energy loss coefficient at the resonant frequency becomes smaller and smaller. It should be noted that, intuitively, this finding seems to be incompatible with that phenomenon shown in Fig. 8a that larger incident wave height leads to smaller amplification of the free-surface elevation in the gap. In fact, these two findings are compatible with each other. The reason lies on that the increase of 


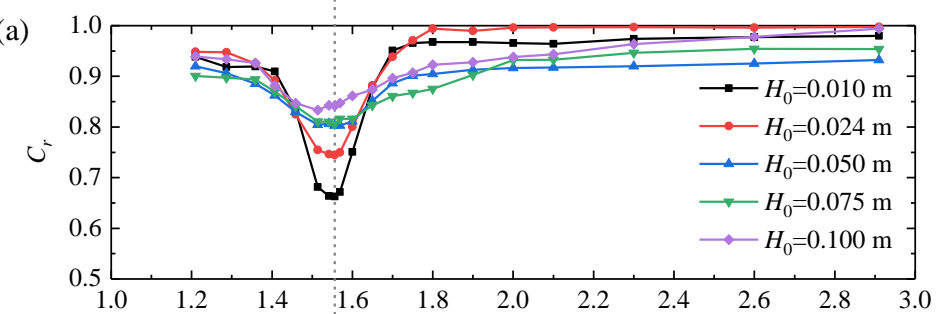

(b)
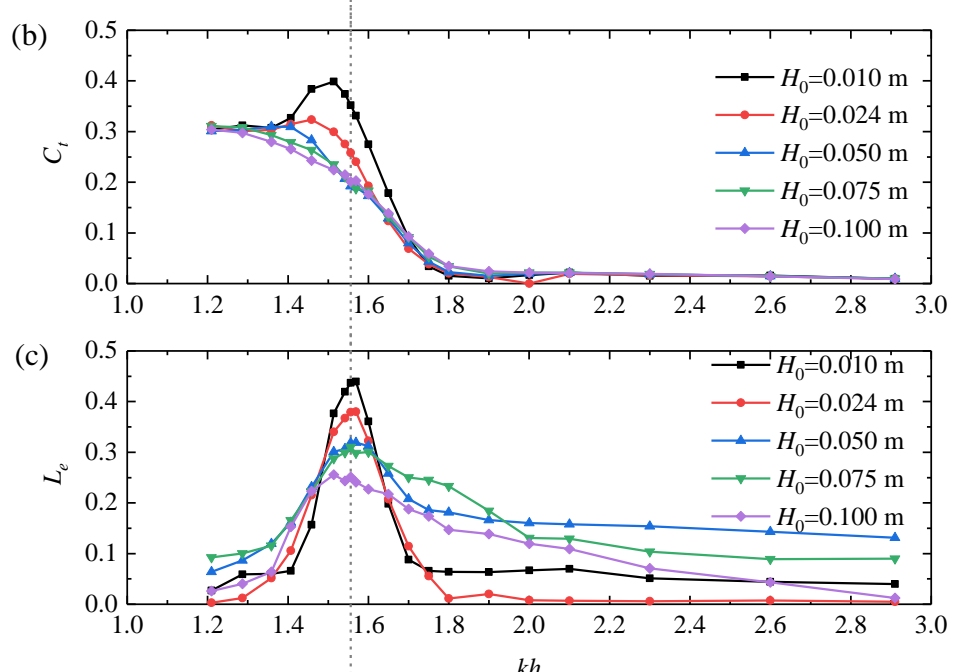

Fig. 18. Variations of (a) the reflection coefficient, (b) the transmission coefficient and (c) the energy loss coefficient with respect to the wave frequency under the conditions of various incident wave heights

\subsection{Response time and damping time of gap resonance}

In practical engineering applications, the fast and accurate estimation of the response time and the damping time is crucial for the safe evacuation of staff and the reasonable arrangement of operation time during the offloading operations from FPSO platforms to a shuttle tanker under gap resonance conditions. In this section, a general method is proposed for accurately estimating both the response time and the damping time of free-surface elevation in the gap under resonance. The general method is based on fitting the envelope of the free-surface elevation. For the evaluation of the response time, there is an alternative method which is based on the amplification curve of the 


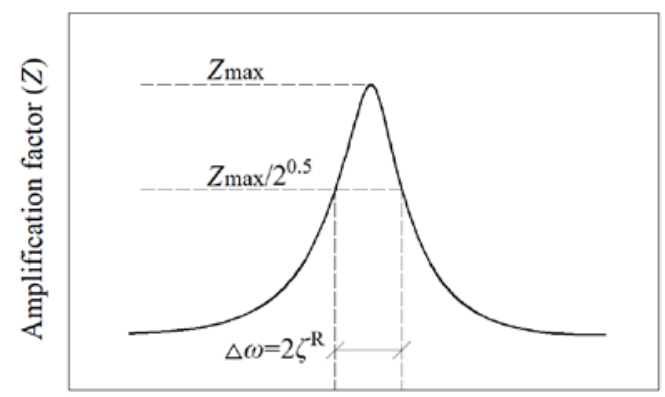

$\omega(\mathrm{rad} / \mathrm{s})$

These methods are inspired by Bellotti (2007) and Dong et al. (2010) who investigated the response time and the damping time of the harbor to long waves under the condition of harbor resonance. In order to facilitate the understanding of the reader, the basic principle of these methods is briefly explained here. To illustrate the basic principle, Fig. 19 shows a generic amplification curve, in which $Z$ is the amplification factor. The free-surface elevation in the narrow gap can be considered as typical of a 1D system like a mass-spring system, moving along a line, connected to a damper, forced by a periodically unit force. If the considered mass starts from rest (i.e., from the position $z=0$ ), when the frequency of the force equals the natural frequency of the system, its position along the axis $(z)$ can be formulated as

$$
z=Z_{\max } \cos \left(-\omega \cdot t^{*}\right)\left(1-e^{-\zeta^{\mathrm{R}} \cdot t^{*}}\right)
$$

in which $\zeta^{\mathrm{R}}$ is a parameter governing the response time of the resonator, $Z_{\max }$ is the maximum amplification factor and $t^{*}$ denotes the relative time with respect to the moment that the mass just begins to move from rest. It requires infinite time for the fluctuation to reach its maximum, following Eq. (7). The time $t_{\alpha \%}^{*}$ needed for the waves to reach $\alpha \%$ of the maximum can be formulated as Similarity, if the mass damps from the steady-state maximum to the rest state, its position can be expressed as

$$
Z=Z_{\max } \cos (-\omega \cdot \tau) e^{-\zeta^{\mathrm{D}} \cdot \tau}
$$

where $\zeta^{\mathrm{D}}$ is a parameter controlling the damping time of the resonator and $\tau$ denotes the relative 
time with respect to the moment that the mass just begins to damp from the steady-state maximum.

The time $\tau_{\beta \%}$ needed by the wave to decrease to $\beta \%$ of the maximum can be expressed as

$$
\tau_{\beta \%}=-\frac{\ln (\beta \%)}{\zeta^{\mathrm{D}}} .
$$

It can be found from Eqs. (8) and (10) that the key step to quantitatively evaluate the response time and the damping time lies on how to find the values of $\zeta^{\mathrm{R}}$ and $\zeta^{\mathrm{D}}$. A general method to obtain their values is to directly fit the measured (or simulated) envelopes of the displacement of the mass with the theoretical ones formulated by Eqs. (7) and (9). It can be demonstrated that for $1 \mathrm{D}$ resonators, the value of $\zeta^{\mathrm{R}}$ can also be evaluated from the amplification curve. More specifically, $\zeta^{\mathrm{R}}=\Delta \omega / 2$, in which $\Delta \omega$ is the half-power spectral bandwidth (i.e., the width of the part of the amplification curve with values larger than $Z_{\max } / 2^{0.5}$ ). Identical to Bellotti (2007) and Dong et al. (2010), $t^{*}{ }_{95 \%}$ and $\tau_{5 \%}$ are selected in this article to represent the response time and the damping time of the resonant free-surface elevations, respectively.
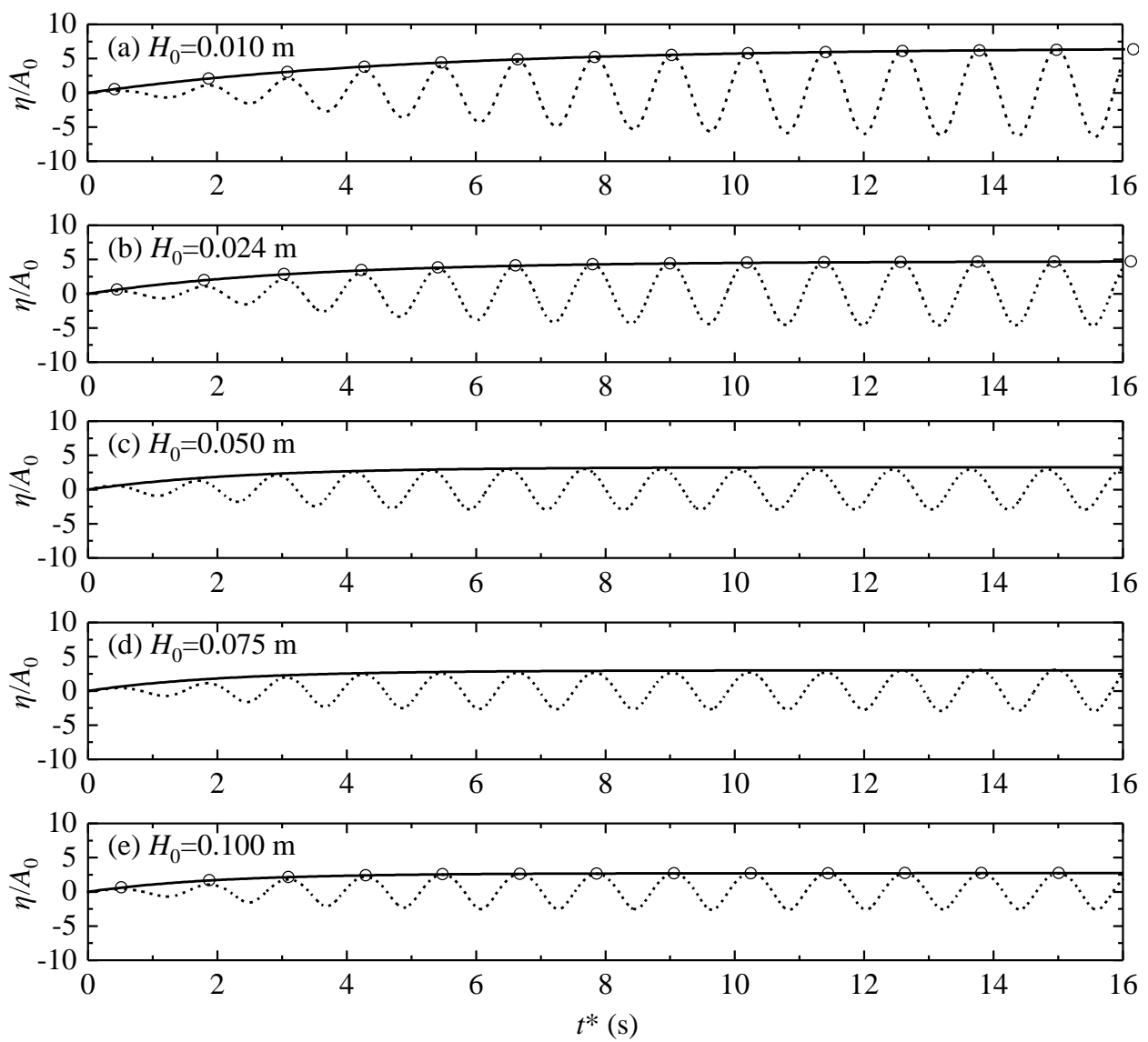

618 Fig. 20. The response process of the free-surface elevation $\left(\eta / A_{0}\right)$ in the narrow gap excited by the 619 incident regular waves with the resonant frequency (i.e., $k h=1.556$, or equivalently $\omega=5.285 \mathrm{rad} / \mathrm{s}$ ) 
and various heights. Dashed lines denote the time histories of the simulated free-surface elevations obtain by the numerical model. Solid lines refer to the fitted envelope of $\eta / A_{0}$ obtained by directly fitting the simulated envelopes with the theoretical ones formulated by Eq. (7). Small circles represent the envelope of $\eta / A_{0}$ as obtained by Eq. (7) using the amplification curve method for estimating $\zeta^{\mathrm{R}}$.

Fig. 20 shows the time histories of the free-surface elevations in the narrow gap, obtained by using the time-resolving numerical model, from the calm to the steady state. The frequency of all the incident regular waves corresponds to the resonant frequency (i.e., $k h=1.556$, or equivalently $\omega=5.285 \mathrm{rad} / \mathrm{s}$ ). By directly fitting the simulated envelopes with the theoretical ones formulated by Eq. (7), the numerical values of $\zeta^{\mathrm{R}}$ can be obtained. Besides, by measuring the half-power spectral bandwidth of the amplification curve as shown in Fig. 19, the values of $\zeta^{\mathrm{R}}$ can also be calculated. It is noted here that, to facilitate comparing the values of $\zeta^{\mathrm{R}}$ obtained by these two different methods, two different symbols, $\zeta_{1}^{\mathrm{R}}$ and $\zeta_{2}^{\mathrm{R}}$, are used separately to represent the values of $\zeta^{\mathrm{R}}$ obtained by the amplification curve method and by the direct envelope-fitting method in the following. As a concrete example of employing the amplification curve method to evaluate the value of $\zeta_{1}^{\mathrm{R}}$, Fig. 21 illustrates the amplification curve of the free-surface elevation in the narrow gap under the condition of $H_{0}=0.010 \mathrm{~m}$. It can be seen that the value of $\zeta_{1}^{\mathrm{R}}$ under the condition of $H_{0}=0.010 \mathrm{~m}$ is equal to 0.20 .

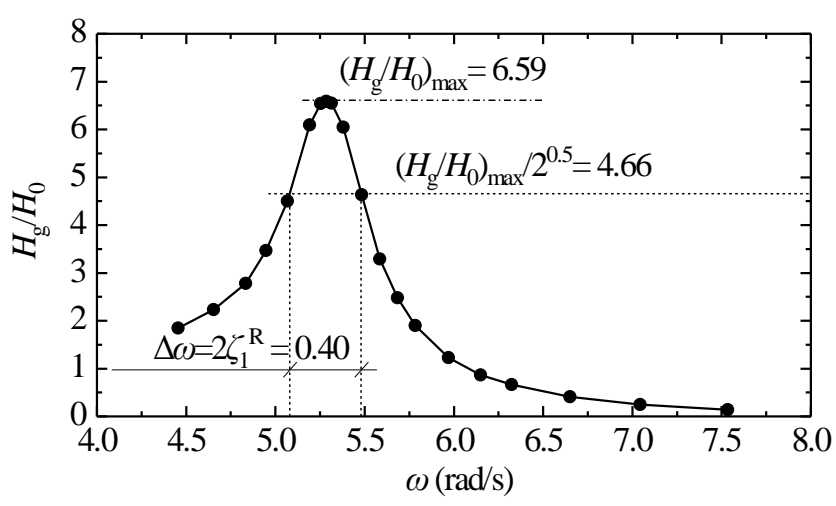

Fig. 21. Amplification curve of the free-surface elevation in the narrow gap under the condition of $H_{0}=0.010 \mathrm{~m}$ 
Table 1 further lists all the values of $\zeta_{1}^{\mathrm{R}}$ and $\zeta_{2}^{\mathrm{R}}$, their relative percentage errors, Err, and the response time, $t^{*}{ }_{95 \%}$ for the free-surface elevations shown in Fig. 20. The response time, $t^{*}{ }_{95 \%}$, in this table is calculated by employing Eq. (8) and the value of $\zeta_{2}^{\mathrm{R}}$. As mentioned in Section 5.1, unlike the typical amplification curves shown in Figs. 19 and 21, the two free-surface amplification curves for $H_{0}=0.050 \mathrm{~m}$ and $0.075 \mathrm{~m}$ do not present the perfect single-peak shape; the two curves around the resonant frequency become flat, and the values of the amplification factor at $k h=1.556$ are even slightly less than the ones at its both adjacent sides (refer to Fig. 8a). Hence, the values of $\zeta_{1}^{\mathrm{R}}$ for $H_{0}=0.050 \mathrm{~m}$ and $0.075 \mathrm{~m}$ are absent. For the other three wave heights, the relative percentage errors between $\zeta_{1}^{\mathrm{R}}$ and $\zeta_{2}^{\mathrm{R}}$ are shown to be extremely small. Besides, observing Fig. 20 can easily find that for all the incident wave heights considered in this paper, both the two envelopes of the free-surface elevations obtained by $\zeta_{1}^{\mathrm{R}}$ and $\zeta_{2}^{\mathrm{R}}$ agree well with the corresponding simulated free-surface elevations by using the time-resolving numerical model. These phenomena indicate that both the two above-mentioned methods for evaluating the response time of gap resonance are accurate and reliable.

Table 1. All the parameters related to the response time and the damping time of the resonant free-surface elevations shown in Figs. 20 and 22. Err denotes the relative percentage error between $\zeta_{1}^{\mathrm{R}}$ and $\zeta_{2}^{\mathrm{R}} \cdot t^{*}{ }_{95 \%}$ and $\tau_{5 \%}$ refers to the response time and the damping time of the 662 free-surface elevations, respectively. The evaluation of $t_{95 \%}^{*}$ is based on Eq. (8) and the value of $\zeta_{2}^{\mathrm{R}}$

\begin{tabular}{cccccccc}
\hline$H_{0}(\mathrm{~m})$ & $\zeta_{1}^{\mathrm{R}}$ & $\zeta_{2}^{\mathrm{R}}$ & $\operatorname{Err}(\%)$ & $t_{95 \%}^{*}(\mathrm{~s})$ & $\zeta^{\mathrm{D}}$ & $\tau_{5 \%}(\mathrm{~s})$ & $\tau_{5 \%} / t_{95 \%}$ \\
\hline 0.010 & 0.200 & 0.202 & 0.99 & 14.83 & 0.125 & 23.97 & 1.62 \\
0.024 & 0.306 & 0.299 & 2.34 & 10.02 & 0.136 & 22.03 & 2.20 \\
0.050 & - & 0.421 & - & 7.12 & 0.145 & 20.66 & 2.91 \\
0.075 & - & 0.467 & - & 6.41 & 0.151 & 19.84 & 3.09 \\
0.100 & 0.512 & 0.499 & 2.60 & 6.00 & 0.158 & 18.96 & 3.16 \\
\hline
\end{tabular}



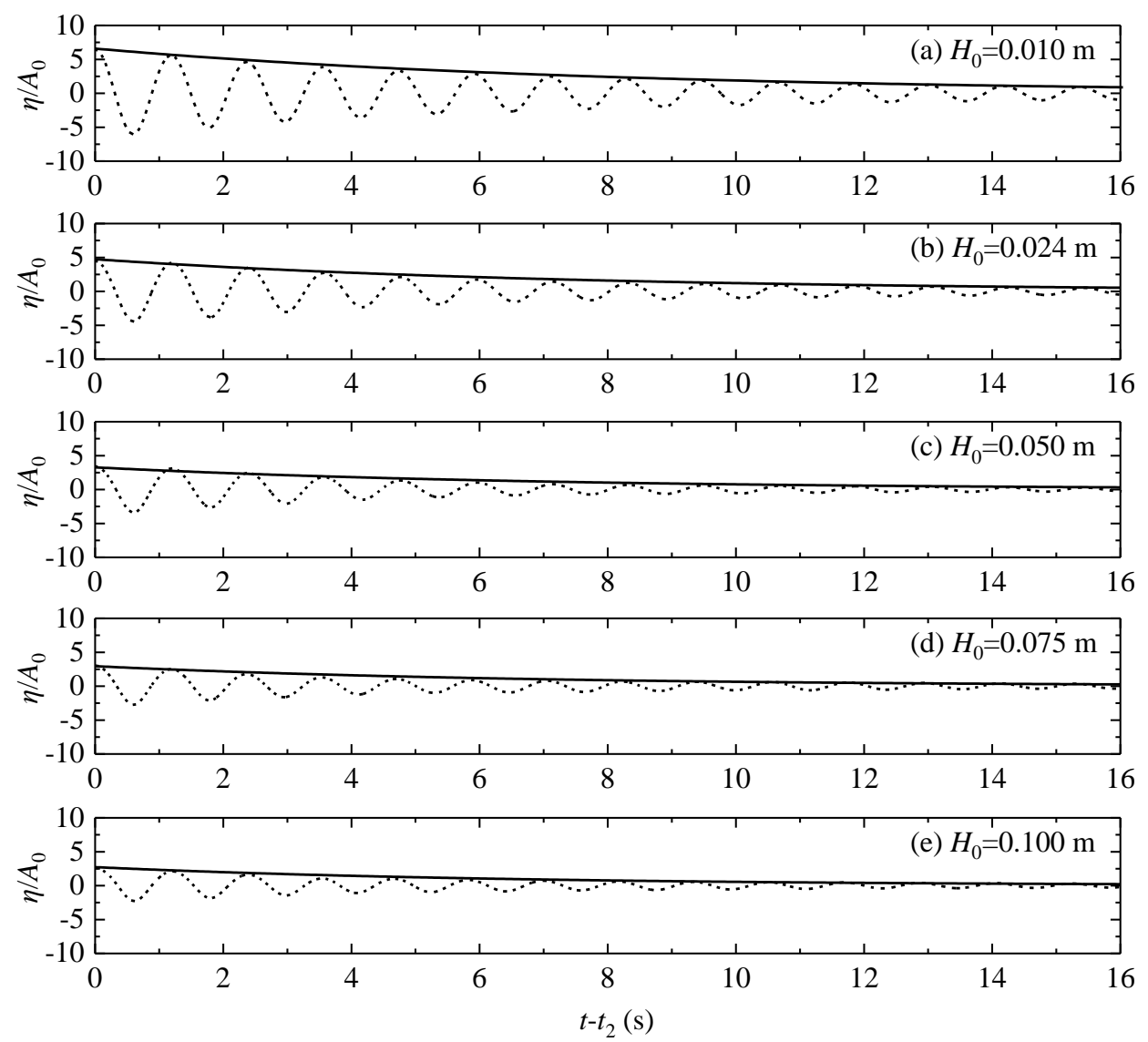

Fig. 22. The damping process of the free-surface elevation $\left(\eta / A_{0}\right)$ in the narrow gap excited by the incident regular waves with the resonant frequency (i.e., $k h=1.556$, or equivalently $\omega=5.285 \mathrm{rad} / \mathrm{s}$ ) and various heights. Dashed lines denote the time histories of the simulated free-surface elevations obtain by the numerical model. Solid lines represent the fitted envelope of $\eta / A_{0}$ obtained by directly fitting the simulated envelopes with the theoretical ones formulated by Eq. (9).

Fig. 22 illustrates the time histories of the simulated free-surface elevations in the narrow gap and their fitted envelopes obtained by directly fitting the simulated envelopes with the theoretical ones formulated by Eq. (9) during their damping processes. It is seen that for all the incident wave heights considered in this paper, Eq. (9) can describe the damping process of the resonant free-surface elevation in the gap very well. All the values of $\zeta^{\mathrm{D}}$ gained by the direct envelope-fitting method and the damping time $\tau_{5 \%}$ under the conditions of various wave heights are also presented in Table 1.

According to the response time and the damping time presented in Table 1, the following two phenomena can be easily observed. First, for all the incident wave heights, the damping time is always significantly larger than the corresponding response time. The ratio of the damping time to 
the response time gradually increases from 1.62 for $H_{0}=0.100 \mathrm{~m}$ to 3.16 for $H_{0}=0.100 \mathrm{~m}$. This indicates that once the gap resonance is excited, it will persist for a rather long time. Second, both the response time and the damping time decrease with the incident wave height, and the decreasing degree of the response time is obviously larger than that of the damping time. The response time falls up to $60 \%$ from $H_{0}=0.010 \mathrm{~m}$ to $H_{0}=0.100 \mathrm{~m}$, while the damping time reduces only about $21 \%$.

\section{Conclusions}

The CFD numerical model, OpenFOAM ${ }^{\circledR}$, together with the wave generation toolbox “waves2Foam” proposed by Jacobsen et al. (2012), is adopted for investigating the hydrodynamic behaviors of water resonance in a narrow gap formed by two side-by-side identical boxes excited by incident regular waves with various wave heights. The overall free-surface amplification in the gap and the overall wave loads on the boxes are firstly presented. Then, the harmonic analyses of free-surface elevation and wave loads are mainly investigated. Next, the reflection, transmission and energy loss coefficients of the two-box system are discussed. Finally, two different methods to evaluate the response time and the damping time of gap resonance are proposed. The results of this study have provided new insights of the hydrodynamic characteristics involved in the gap resonance.

The following conclusions can be drawn from the results of the present study:

(1) The frequencies at which the maximum vertical wave forces on both boxes and the maximum horizontal wave force on Box A occur appear to obviously deviate from the resonant frequency, and a larger incident wave height tends to cause more obvious differences between them. While the frequency at which the maximum horizontal force on Box B occurs is equal or very close to the resonant frequency.

(2) For the free-surface elevation in the gap and the moments on boxes, the ratios of their second-order components to the corresponding first-order ones around the resonant frequency are normally larger than those at the frequencies far from the resonant frequency (except those at the very high frequency band). The larger the incident wave height is, the larger the ratios of the second- to the first-order components around the resonant frequency becomes.

(3) For both the vertical and horizontal wave forces on both boxes, the ratios of their second- to 
the first-order components near the resonant frequency are less than those far away from the resonant frequency. Besides, when the incident wave height is small, their second-order components are obviously larger than the corresponding third-order ones around the resonant

(4) Both the minimum reflection coefficient and the maximum energy loss coefficient always

\section{Acknowledgments}

This work is financially supported by the National Natural Science Foundation of China (Grant no. 51609108) and the Jiangsu Government Scholarship for Overseas Studies (awarded to Dr. Junliang Gao for study abroad at the University of Bath). The authors also thank UK EPSRC and the Royal Academy of Engineering for providing partial support for this work within the UK-China joint projects ResIn (EPSRC Grant No. EP/R007519/1) and the UK-CIAPP (RAE 
Grant No. UK-CIAPP/73). We also express our thanks to Dr. Wei Bai (Manchester Metropolitan University) and Dr. Xingya Feng (University of Oxford) for their support for this study.

\section{References}

Bellotti, G., 2007. Transient response of harbours to long waves under resonance conditions. Coastal Engineering 54 (9), 680-693.

Chen, X.B., 2004. Hydrodynamics in Offshore and Naval Applications (Keynote lecture), The 6th International Conference on Hydrodynamics, Perth, Austrilia.

Dong, G., Wang, G., Ma, X., Ma, Y., 2010. Numerical study of transient nonlinear harbor resonance. Science China-Technological Sciences 53, 558-565. 
Engineering 117, 210-220.

Lu, L., Chen, L., Teng, B., Zhao, M., 2010a. Numerical investigation of fluid resonance in two narrow gaps of three identical rectangular structures. Applied Ocean Research 32 177-190.

Lu, L., Cheng, L., Teng, B., Sun, L., 2010b. Numerical simulation and comparison of potential flow and viscous fluid models in near trapping of narrow gaps. Journal of Hydrodynamics, Ser. B 22 (5), 120-125.

Lu, L., Teng, B., Cheng, L., Li, Y., 2008. Numerical simulation of hydrodynamic resonance in a narrow gap between twin bodies subject to water waves, The Eighteenth International Offshore and Polar Engineering Conference, Vancouver, Canada.

Lu, L., Teng, B., Cheng, L., Sun, L., Chen, X., 2011a. Modelling of multi-bodies in close proximity under water waves-Fluid resonance in narrow gaps. Science China Physics, Mechanics and Astronomy 54 (1), 16-25.

Lu, L., Teng, B., Sun, L., Chen, B., 2011b. Modelling of multi-bodies in close proximity under water waves—Fluid forces on floating bodies. Ocean Engineering 38 (13), 1403-1416.

Mavrakos, S.A., Chatjigeorgiou, I.K., 2009. Second-order hydrodynamic effects on an arrangement of two concentric truncated vertical cylinders. Marine Structures 22 (3), 545-575.

Miao, G., Ishida, H., Saitoh, T., 2000. Influence of gaps between multiple floating bodies on wave forces. China Ocean Engineering 14 (4), 407-422.

Molin, B., 2001. On the piston and sloshing modes in moonpools. Journal of Fluid Mechanics 430, 27-50.

Moradi, N., Zhou, T., Cheng, L., 2015. Effect of inlet configuration on wave resonance in the narrow gap of two fixed bodies in close proximity. Ocean Engineering 103 88-102.

Moradi, N., Zhou, T., Cheng, L., 2016. Two-dimensional numerical study on the effect of water depth on resonance behaviour of the fluid trapped between two side-by-side bodies. Applied Ocean Research 58, 218-231.

Newman, J.N., 2004. Progress in wave load computations on offshore structures (Invited lecture), The 23th Conference on Offshore Mechanics and Arctic Engineering (OMAE2004), New York, USA.

Ning, D., Su, X., Zhao, M., Teng, B., 2015a. Hydrodynamic difference of rectangular-box systems 
with and without narrow gaps. Journal of Engineering Mechanics 141 (8), 04015023.

Ning, D., Su, X., Zhao, M., Teng, B., 2015b. Numerical study of resonance induced by wave action on multiple rectangular boxes with narrow gaps. Acta Oceanologica Sinica 34 (5), 92-102.

Pauw, W.H., H.M.Huijsmans, R., Voogt, A., 2007. Advances in the Hydrodynamics of Side-by-Side Moored Vessels, Proceedings of the 26th International Conference on Offshore Mechanics and Arctic Engineering (OMAE2017), San Diego, California, USA.

Rodríguez, M., Spinneken, J., 2016. A laboratory study on the loading and motion of a heaving box. Journal of Fluids and Structures 64, 107-126.

Saitoh, T., Miao, G., Ishida, H., 2006. Theoretical analysis on appearance condition of fluid resonance in a narrow gap between two modules of very large floating structure, Proceedings of the 3rd Asia-Pacific Workshop on Marine Hydrodynamics, Shanghai, China, pp. 170-175.

Sun, L., Taylor, R.E., Taylor, P.H., 2010. First- and second-order analysis of resonant waves between adjacent barges. Journal of Fluids and Structures 26 (6), 954-978.

Tan, L., Lu, L., Liu, Y., Sabodash, O.A., Teng, B., 2014. Dissipative Effects of Resonant Waves in Confined Space Formed by Floating Box in Front of Vertical Wall, The Eleventh ISOPE Pacific/Asia Offshore Mechanics Symposium, Shanghai, China 